OPEN ACCESS

Edited by:

Tonya J. Webb,

University of Maryland, Baltimore,

United States

Reviewed by:

Xueqiong Zhu,

Wenzhou Medical University, China

Matteo Morotti,

Centre Hospitalier Universitaire

Vaudois (CHUV), Switzerland

${ }^{*}$ Correspondence:

Michael J. Birrer

mibirrer@uams.edu

Specialty section:

This article was submitted to

Gynecological Oncology,

a section of the journal

Frontiers in Oncology

Received: 12 November 2021

Accepted: 24 January 2022

Published: 23 February 2022

Citation:

Birrer MJ, Fujiwara K, Oaknin A Randall L, Ojalvo LS, Valencia C and Ray-Coquard I (2022) The Changing Landscape of Systemic Treatment

for Cervical Cancer: Rationale

for Inhibition of the TGF- $\beta$

and PD-L1 Pathways.

Front. Oncol. 12:814169.

doi: 10.3389/fonc.2022.814169

\section{The Changing Landscape of Systemic Treatment for Cervical Cancer: Rationale for Inhibition of the TGF- $\beta$ and PD-L1 Pathways}

\author{
Michael J. Birrer ${ }^{1 *}$, Keiichi Fujiwara ${ }^{2}$, Ana Oaknin $^{3}$, Leslie Randall ${ }^{4}$, Laureen S. Ojalvo ${ }^{5}$, \\ Christian Valencia ${ }^{5}$ and Isabelle Ray-Coquard ${ }^{6}$ \\ 1 Winthrop P. Rockefeller Cancer Institute, University of Arkansas Medical School, Little Rock, AR, United States, \\ 2 Department of Gynecologic Oncology, Saitama Medical University International Medical Center, Saitama, Japan, \\ ${ }^{3}$ Gynaecological Cancer Program, Vall d'Hebron Institute of Oncology, Vall d'Hebron University Hospital, Barcelona, Spain, \\ ${ }^{4}$ Massey Cancer Center, Virginia Commonwealth University, Richmond, VA, United States, ${ }^{5}$ EMD Serono Research \& \\ Development Institute, Inc., Billerica, MA, United States, ${ }^{6}$ GINECO Group \& Department of Medical Oncology, Centre Leon \\ Berard, University Claude Bernard Lyon, Lyon, France
}

Cervical cancer is one of the most common and lethal cancers among women worldwide. Treatment options are limited in patients with persistent, recurrent, or metastatic cervical cancer, with $<20 \%$ of women living $>5$ years. Persistent human papillomavirus (HPV) infection has been implicated in almost all cases of cervical cancer. HPV infection not only causes normal cervical cells to transform into cancer cells, but also creates an immunosuppressive environment for cancer cells to evade the immune system. Recent clinical trials of drugs targeting the PD-(L)1 pathway have demonstrated improvement in overall survival in patients with cervical cancer, but only $20 \%$ to $30 \%$ of patients show overall survival benefit beyond 2 years, and resistance to these treatments remains common. Therefore, novel treatment strategies targeting HPV infection-associated factors are currently being evaluated in clinical trials. Bintrafusp alfa is a first-in-class bifunctional fusion protein composed of the extracellular domain of the TGF- $\beta$ RII receptor (a TGF- $\beta$ "trap") fused to a human immunoglobulin G1 monoclonal antibody that blocks PD-L1. Early clinical trials of bintrafusp alfa have shown promising results in patients with advanced cervical cancer.

Keywords: cervical cancer, HPV, tumor microenvironment, TGF- $\beta$, PD-L1

\section{INTRODUCTION}

With an estimated 604,000 new cases and 342,000 deaths globally in 2020, cervical cancer (malignant neoplasm of cervix uteri) is the fourth most commonly diagnosed cancer and the fourth leading cause of cancer-related death among women worldwide (1). In women younger than 45 years, cervical cancer was among the top 3 most common cancers in $79 \%$ of countries based on 2018 estimates (2) (Figure 1). 


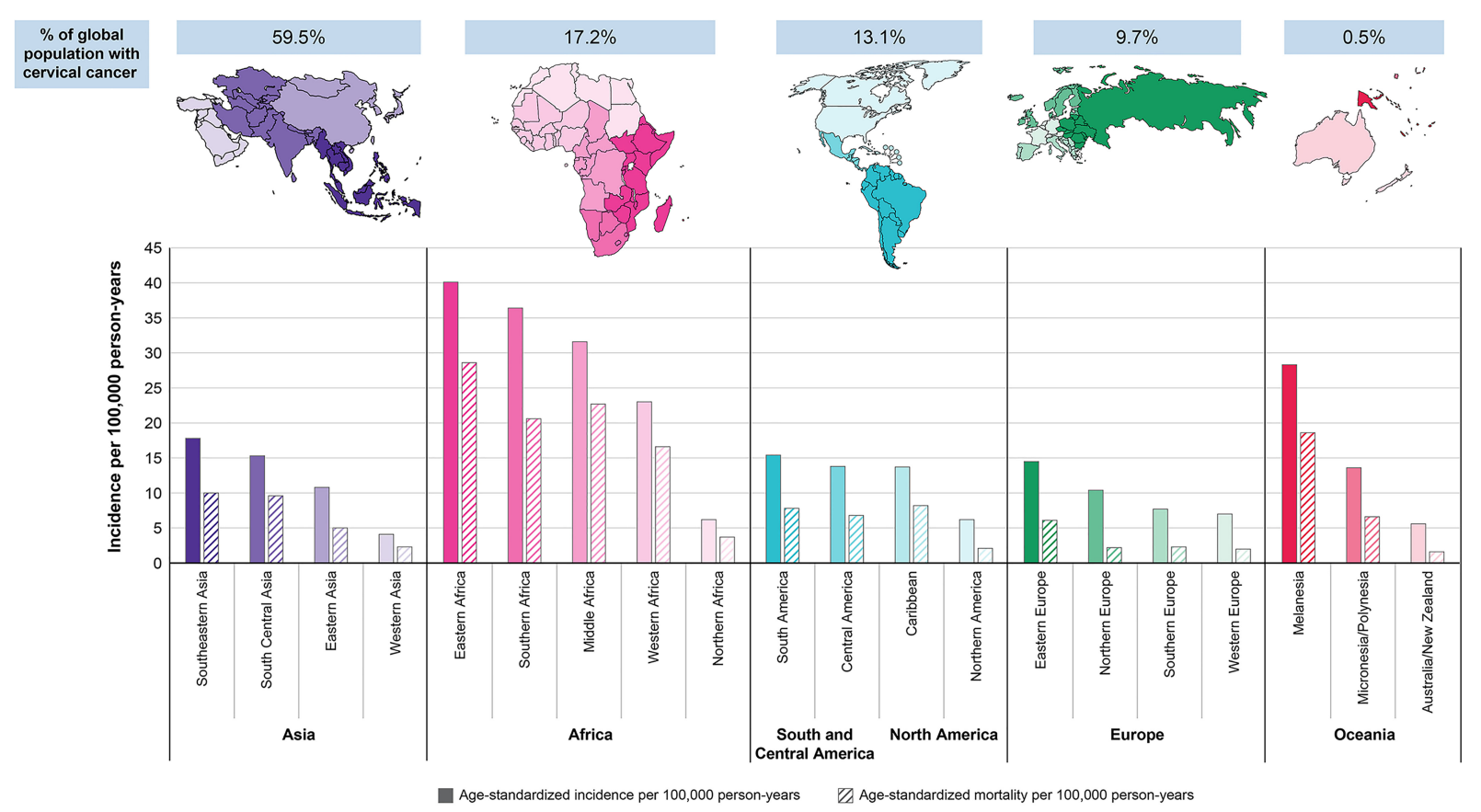

FIGURE 1 | Region-specific age-standardized incidence and mortality rates for cervical cancer in 2020 (1).

Cervical cancer is divided into 2 main histological subtypes: squamous cell carcinoma, which accounts for $70 \%$ to $80 \%$ of cervical cancers, and adenocarcinoma, which accounts for $20 \%$ to $25 \%(3,4)$. Most data suggest that the prognosis of adenocarcinomas is worse than that of squamous cell carcinomas (3); however, these subtypes are treated similarly in the persistent, recurrent, or metastatic setting.

Cancer stage at diagnosis determines treatment options and influences the length of survival (5). Rates vary globally, with $12 \%$ to $36 \%$ of patients diagnosed with regional (stage II/III) disease and $5 \%$ to $16 \%$ diagnosed with distant or metastatic (stage IV) disease in the US, EU, and Japan (5-8). Among lowincome and lower middle-income countries, cervical cancer is commonly diagnosed in the later stages of disease, most likely due to poor accessibility to preventive care $(9,10)$. Early cervical cancers and precancers usually do not cause noticeable signs or symptoms, which contributes to patients being diagnosed at a later stage (11). However, patients with recurrent or metastatic cervical cancer may have a variety of symptoms, including pain, anorexia, vaginal bleeding, cachexia, and psychological problems (12).

Women with unresectable, locally advanced cervical cancer are frequently treated using chemoradiotherapy (CRT); however, CRT is associated with late toxicities that impact quality of life, and recurrence after CRT carries a very poor prognosis (13-16). While advancements in CRT have improved overall survival (OS) in these patients, the risk of recurrence, typically within 2 years of diagnosis, is significant $(3,16,17)$. In addition, treatment-related urinary, gastrointestinal, sexual, and neurological adverse effects can disrupt long-term quality of life (18). Survivors can also experience persistent emotional and psychological distress (19).

Persistent human papillomavirus (HPV) infection plays a crucial role in the pathogenesis of cervical cancers, particularly the oncogenic subtypes HPV16 and HPV18, which are responsible for $70 \%$ of cervical cancers (3, 10, 20-23). Cervical cancer cases occur at higher rates in countries that have not introduced an HPV vaccination program than in those that have implemented a vaccination program $(10,21)$. According to the World Health Organization's Cervical Cancer Elimination Modelling Consortium, high HPV vaccination coverage has the potential to reduce cervical cancer mortality by almost $99 \%$ in most low-income and lower middle-income countries by the end of the century (9). Until then, understanding the biological role of HPV infection may be the key to identifying new treatments.

HPV infection mediates cervical cancer pathogenesis through various pathways. During persistent infection, viral DNA is randomly integrated into the host genome, leading to cellular immortalization and eventually to malignant progression (24). The combined action of HPV oncoproteins E6 and E7 can mediate the transformation of cervical cells into cancerous cells by driving cell proliferation, interfering with apoptosis, and promoting genomic instability; their expression is required to maintain a proliferative and malignant phenotype (24). Chronic immunosuppression carries a higher risk for malignant transformation with persistent HPV infection (25).

Here, we discuss the current treatment options and the future of systemic treatment for patients with persistent (defined as 
cervical cancer that remains present after treatment for locally advanced disease), recurrent (defined as cervical cancer that has progressed after treatment for locally advanced disease), or metastatic cervical cancer. We focus on the role of HPV infection, which has been implicated in $99 \%$ of all cases of cervical cancer, in the underlying disease biology of cervical cancer (3).

\section{CURRENT TREATMENT FOR PERSISTENT, RECURRENT, OR METASTATIC CERVICAL CANCER}

Chemotherapy doublets, primarily platinum based, were the first-line standard of care in the persistent, recurrent, or metastatic setting for many years (3). While chemotherapy doublets may still be used, adding bevacizumab, an antivascular endothelial growth factor (VEGF) monoclonal antibody, to cisplatin-paclitaxel is the preferred first-line regimen for patients with persistent, recurrent, or metastatic cervical cancer $(3,26,27)$. The preference for this regimen was based on the results of the phase 3 GOG 240 randomized trial, which showed that the addition of bevacizumab to a chemotherapy doublet (cisplatin-paclitaxel or topotecanpaclitaxel) significantly improved median OS (16.8 vs 13.3 months; $\mathrm{p}=0.0068$ ) and median progression-free survival (PFS; 8.2 vs 6.0 months; $P=0.0002$ ) compared with a chemotherapy doublet alone $(3,26,28,29)$. Although the OS difference between bevacizumab plus topotecan-paclitaxel and topotecan-paclitaxel was not statistically significant, this regimen is considered an alternative to cisplatin-paclitaxel plus bevacizumab in patients who are not eligible for cisplatin (27-30). The addition of bevacizumab to the chemotherapy doublet increased the incidence of grade $\geq 3$ thromboembolic events, grade $\geq 2$ hypertension, and fistulae occurrence, warranting more vigilant patient monitoring $(3,29)$. Carboplatin is routinely used instead of cisplatin in some countries and for specific patient subpopulations, such as those with renal impairment and those who have experienced relapse after prior cisplatin $(31,32)$. The use of carboplatin either as part of a doublet or with bevacizumab was evaluated in two trials [phase 3 JCOG0505 trial (31) and phase 2 CECILIA trial (32)]. Based on these data, carboplatin in combination with bevacizumab and paclitaxel is also considered an alternative regimen $(26,31,32)$.

Despite the current best treatment of platinum-based doublet chemotherapy with bevacizumab, data from the GOG 240 trial showed a median PFS of 8.2 months in patients with persistent, recurrent, or metastatic cervical cancer, and $>60 \%$ had disease progression within 12 months (29). In addition, median OS was 16.8 months, and the 5 -year OS rates were $<20 \%$, irrespective of the chemotherapy used (29).

Recently, the US Food and Drug Administration (FDA) approved pembrolizumab in combination with chemotherapy, with or without bevacizumab, as first-line treatment for patients with persistent, recurrent, or metastatic cervical cancer whose tumors express programmed death ligand 1 (PD-L1; combined positive score $[\mathrm{CPS}] \geq 1$ ) (33). This approval was based on the phase 3 KEYNOTE-826 trial, in which median PFS with pembrolizumab vs placebo was 10.4 vs 8.2 months (hazard ratio, $0.62 ; 95 \% \mathrm{CI}, 0.50-0.77 ; P<0.001)$ and median $\mathrm{OS}$ was not reached vs 16.3 months (hazard ratio, $0.64 ; 95 \%$ CI, 0.50 $0.81 ; P=0.0001)(34,35)$. Median duration of response (DOR) with pembrolizumab vs placebo was 18.0 vs 10.4 months and objective response rate (ORR) was $68.1 \%$ vs $50.2 \%$.

No standard-of-care treatment for patients with recurrent or metastatic cervical cancer that progresses during or after platinum-containing chemotherapy is currently globally accepted (3, 26). Single-agent chemotherapy has limited efficacy in the second-line setting, with ORRs often $\leq 15 \%$, median PFS $<6$ months, and median OS $<10$ months (3). Patients with recurrent or metastatic cervical cancer who experience disease progression on or after chemotherapy and whose tumors express PD-L1 (CPS $\geq 1$ ) may receive pembrolizumab based on approval by the US FDA $(33,34,36)$; however, there have been no approvals outside of the US to date. This accelerated approval was based on an ORR of $14.3 \%$ and a median DOR that was not reached (range, 4.1-18.6+ months) in the single-arm, phase 2 KEYNOTE-158 study (37). In an updated analysis with a median follow-up of 36.9 months, the ORR was $17.1 \%$ in the PD-L1-positive cohort with median DOR still not reached (38). Patients in the study with PD-L1-negative tumors did not respond to pembrolizumab. Immune-mediated adverse events (AEs), common with checkpoint inhibitor therapy, occurred in 25 patients $(25.5 \%)$, with five patients (5.1\%) experiencing one or more grade $3 / 4$ events (37). No treatment-related deaths or immune-mediated AEs leading to death were reported. These data indicate the potential benefit of inhibiting PD-L1 but also the challenges in providing benefit to patients whose tumors do not express PD-L1. Promising results have also been reported recently with cemiplimab in second-line or later settings in patients with persistent, recurrent, or metastatic cervical cancer (39).

Given these data, while some advances in treatment have been made with anti-VEGF inhibitors and immune checkpoint inhibitors, much room exists for improving outcomes in patients with persistent, recurrent, or metastatic cervical cancer. Thus, a rational approach to identify new systemic therapies that address the underlying pathophysiology of the disease is needed.

\section{THE ROLE OF TRANSFORMING GROWTH FACTOR $\beta$ (TGF- $\beta$ ) AND PD-L1 IN CERVICAL CANCER}

HPV infections that persist and progress to cervical cancer evade the immune system, in part by creating an immunosuppressive environment (40-44). During HPV-associated cervical cancer progression, $\mathrm{T}$ helper 1 cytokines such as interferon- $\gamma$ and interleukin (IL)-2 are downregulated, and T helper 2 cytokines such as TGF- $\beta$ and IL-10 are upregulated; these cytokine changes produce a local immunosuppressive tumor microenvironment 
(TME) that inhibits antitumor immune responses (42-44). Genes responsible for promoting HPV gene expression, known as master regulators, are also associated with expression of molecules such as PD-L1, TGF- $\beta$, and IL-10, which also promote an immunosuppressive environment (45). Increases in programmed death 1 (PD-1) and PD-L1 (PD-[L]1) expression have been positively associated with HPV infection, increase in cervical intraepithelial neoplasia grade, and tumor metastasis in patients with cervical cancer (46).

Preclinical studies have shown that HPV oncoproteins E6 and E7 can upregulate TGF- $\beta 1$ promoter activity (47) and can increase expression of immunosuppressive cytokines, resulting in overexpression of TGF- $\beta$, which stimulates survival and proliferation of cervical cancer cell lines $(44,48,49)$. Aberrant TGF- $\beta$ activation and signaling can promote tumorigenesis by stimulating epithelial-mesenchymal transition (EMT), angiogenesis, cancer-associated fibroblast activation, and immunosuppression within the TME (42, 50-52) (Figure 2). EMT has been implicated in cervical cancer and is shown to be associated with tumor progression and metastasis of primary tumors (53-56). TGF- $\beta$-mediated signaling in the TME upregulates the expression of VEGF, which promotes angiogenesis, facilitating nutrient exchange and metastasis that results in tumor progression $(57,58)$. TGF- $\beta$ is a well-known regulator of fibrosis and can recruit cancer-associated fibroblasts to the TME and promote extracellular matrix remodeling, which has been implicated in increased cancer invasion, metastasis, and resistance to anticancer therapy in preclinical studies (59-67).

A positive correlation between HPV infection and TGF- $\beta$ expression was observed in the serum and saliva of patients with oral squamous cell carcinoma, a cancer type also known to be associated with HPV infection (68). RNA sequencing analysis of HPV-positive oropharyngeal squamous cell carcinoma showed that high E6 expression was associated with poor survival (69). Hence, dysregulation of the TGF- $\beta$ pathway due to HPV infection may play a critical role in carcinogenesis, not only in cervical cancer, but also in other HPV-associated cancers.

Increased TGF- $\beta$ activity has also been implicated in resistance to immunotherapy in urothelial and colorectal tumors $(70,71)$. Elevated TGF- $\beta$ levels in the TME represent a primary mechanism of immune evasion, which may play a role in mediating resistance to existing therapies targeting the PD-(L) 1 pathway $(42,50,72-77)$. Inhibition of TGF- $\beta$ has been shown to enhance the response to PD-(L)1 blockade in preclinical studies by priming the TME and promoting T-cell infiltration (78). Furthermore, current standard-of-care

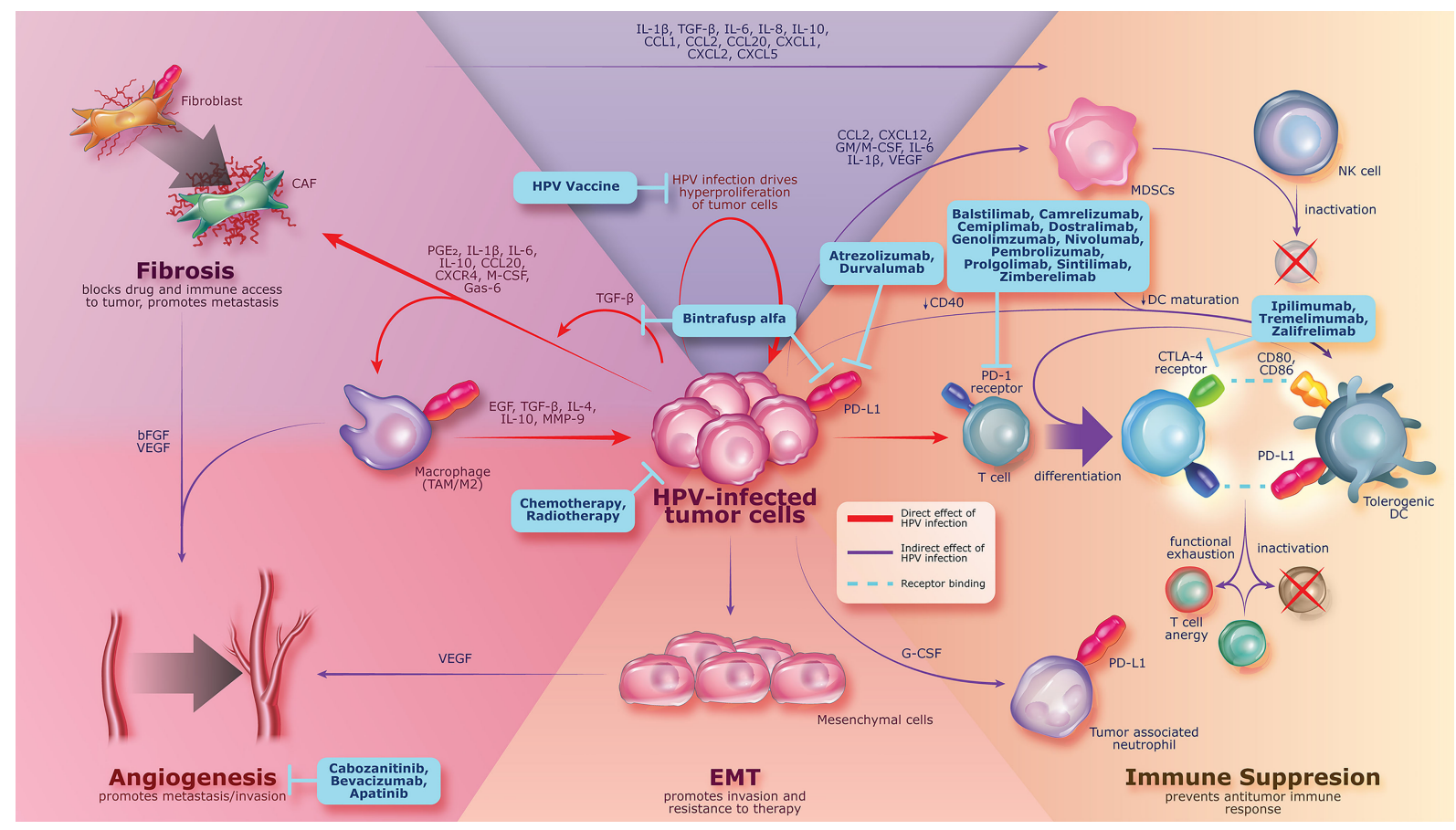

FIGURE 2 | Persistent HPV infection leading to pathogenesis of cervical cancer by increasing the expression of immunosuppressive proteins (e.g., TGF- $\beta$, PD-1, and PD-L1), which can promote tumorigenesis, angiogenesis, fibrosis, and immune suppression. Current treatment strategies are shown in blue boxes and include agents with activity against cancer-associated changes related to HPV infection. bFGF, basic fibroblast growth factor; CAF, cancer-associated fibroblast; CCL, chemokine C-C motif ligand; CD, cluster of differentiation; CTLA-4, cytotoxic T-lymphocyte protein 4; CXCL, chemokine C-X-C motif ligand; DC, dendritic cell; EGF, epidermal growth factor; EMT, epithelial-mesenchymal transition; Gas, growth arrest-specific protein; G/M-CSF, granulocyte/macrophage colony-stimulating factor; HPV, human papillomavirus; IL, interleukin; MDSC, myeloid-derived suppressor cell; MMP, matrix metallopeptidase; NK, natural killer; PD-1, programmed death 1; PD-L1, programmed death ligand 1 ; PGE 2 , prostaglandin $E_{2}$; TAM, tumor-associated macrophage; TGF- $\beta$, transforming growth factor $\beta$; VEGF, vascular endothelial growth factor. 
therapies, including radiotherapy and chemotherapy, have been demonstrated to upregulate the TGF- $\beta$ and PD-(L) 1 pathways (79-82). In various mouse models of human cancer and in patient samples, radiation therapy and chemotherapy increase TGF- $\beta$ signaling, expression, and protein secretion $(79,80,83$, $84)$. In addition, these treatments upregulate PD-1 expression on immune cells and PD-L1 expression on tumor cells, which could potentially mediate treatment resistance $(81,82,85,86)$. The important roles of TGF- $\beta$ and PD-L1 in the development and progression of cervical cancer support the concept that these pathways are rational therapeutic targets.

\section{FUTURE TREATMENT OPTIONS IN CERVICAL CANCER TARGETING HPV-MEDIATED PATHWAYS}

\section{First-Line Therapy}

While many novel strategies that address the biology of cervical cancer are being explored with the aim of improving outcomes in the first-line setting (Figure 2), agents with activity against cancer-associated changes related to HPV infection are a key focus. These include anti-PD-(L) 1 therapies, HPV therapeutic vaccines, and the bifunctional fusion protein bintrafusp alfa.

Based on the efficacy demonstrated with pembrolizumab and the significant role of PD-L1 in cervical cancer, various anti-PD-(L)1 agents are currently under investigation as firstline therapies in cervical cancer (Tables 1 and 2). Phase 3 trials of atezolizumab and prolgolimab (BCD-100) in combination with chemotherapy with or without bevacizumab and pembrolizumab with chemoradiotherapy are ongoing (Table 2).

In addition, several trials are examining novel combinations. One such novel combination includes the HPV vaccine ISA101, which initiates an immune response to HPV16 proteins E6 and E7 and presumably results in cells infected with HPV16 being recognized by the immune system (84). ISA101 in combination with chemotherapy has shown promising results in patients with advanced cervical cancer $(88,112)$. This vaccine is injected after initiation of chemotherapy at a time point when reduction in myeloid cells leads to decreased immunosuppression and a strong T-cell response to the ISA101 vaccine. Median OS was 16.8 months in 32 patients with a high HPV16-specific vaccineinduced immune response compared with 11.2 months in 32 patients with a low HPV16-specific vaccine-induced immune response in an open-label phase $1 / 2$ study in patients with HPVpositive cervical cancer (88). Additionally, 31 of 72 patients (43\%) had tumor regression; however, given that this was a single-arm study, it was not possible to assess whether antitumor activity was due to the impact of ISA101 plus chemotherapy or chemotherapy alone.

\section{Post Platinum-Based Therapy}

The potential of anti-PD-(L)1 therapies in second and later lines of therapy for cervical cancer was demonstrated with the approval of pembrolizumab for patients with recurrent or metastatic cervical cancer who experience disease progression on or after chemotherapy and whose tumors express PD-L1. However, an unmet need remains because response rates with pembrolizumab were low and treatment eligibility is restricted based on PD-L1 expression. Several other anti-PD-(L)1 treatments and novel agents are being explored in this setting.

\section{Monotherapies}

Anti-PD-(L)1 therapies such as atezolizumab, durvalumab, nivolumab, and pembrolizumab have demonstrated strong efficacy in other tumor types but have limited efficacy as second-line treatment in cervical cancer, although safety profiles are consistent across tumor types. In small phase $1 / 2$ trials in patients with cervical cancer, balstilimab, cemiplimab, nivolumab, pembrolizumab, and zimberelimab monotherapies have reported ORRs from $4 \%$ to $26.8 \%$, and while median DORs were not reached in many trials, the median OS was approximately 1 year with most agents (Table 1).

Recently reported data from the phase 3 EMPOWER trial of cemiplimab, an anti-PD-1 agent, in patients with recurrent, persistent, or metastatic cervical cancer who had disease progression with prior platinum-based chemotherapy have for the first time showed that anti-PD-(L) 1 therapy can produce better outcomes than chemotherapy in this setting (39). This trial evaluated cemiplimab monotherapy vs investigator choice of single-agent chemotherapy; all patients had received one prior line of therapy, and $40.8 \%$ had received more than one prior therapy. In the overall patient population, an improvement in median OS from 8.5 months with chemotherapy to 12.0 months with cemiplimab $(P<0.001)$ was reported, and the trial was stopped early $(39,96,113)$. Median PFS was 2.8 months with cemiplimab and 2.9 months with chemotherapy (hazard ratio, 0.75; 95\% CI, 0.63-0.89; $P=0.00048$ ); ORRs were $16.4 \%$ and $6.3 \%$, respectively. Treatment-related AEs occurred in $56.7 \%$ of patients receiving cemiplimab, including $14.7 \%$ with grade $3 / 4$ events; the most common treatment-emergent AEs were anemia (25.0\%), nausea (18.3\%), and fatigue (16.7\%) (39). However, this study did not select patients by PD-L1 status, and therefore assessment by PD-L1 subgroup is not available.

In the EMPOWER trial, cemiplimab resulted in a higher ORR in patients with squamous cell carcinoma than in those with adenocarcinoma (17.6\% vs $12.3 \%)$. These results support a general trend that response rates in patients with adenocarcinoma are typically lower than those in patients with squamous cell carcinoma $(3,39,114)$. This has been particularly evident with anti-PD-(L)1 therapies in the second- or later-line setting. In the NRG-GY002 trial, nivolumab treatment resulted in one partial response in 15 patients $(6.7 \%)$ with squamous cell carcinoma, but no responses occurred in the six patients with adenocarcinoma (115). In a phase 2 study with balstilimab monotherapy in patients with at least one prior chemotherapy, responses were seen in 15 of 85 patients $(17.6 \%)$ with squamous cell carcinoma and in six of 48 (12.5\%) with adenocarcinoma (93). In the KEYNOTE-158 trial, only one of five patients with adenocarcinoma had a response, making it difficult to draw any conclusions; 11 of 92 patients (12\%) with squamous cell carcinoma had a response (37). Median OS was improved with cemiplimab in both histological types compared with single- 
TABLE 1 | Efficacy and safety of immunotherapy agents as monotherapy or in combination with other therapies in cervical cancers.

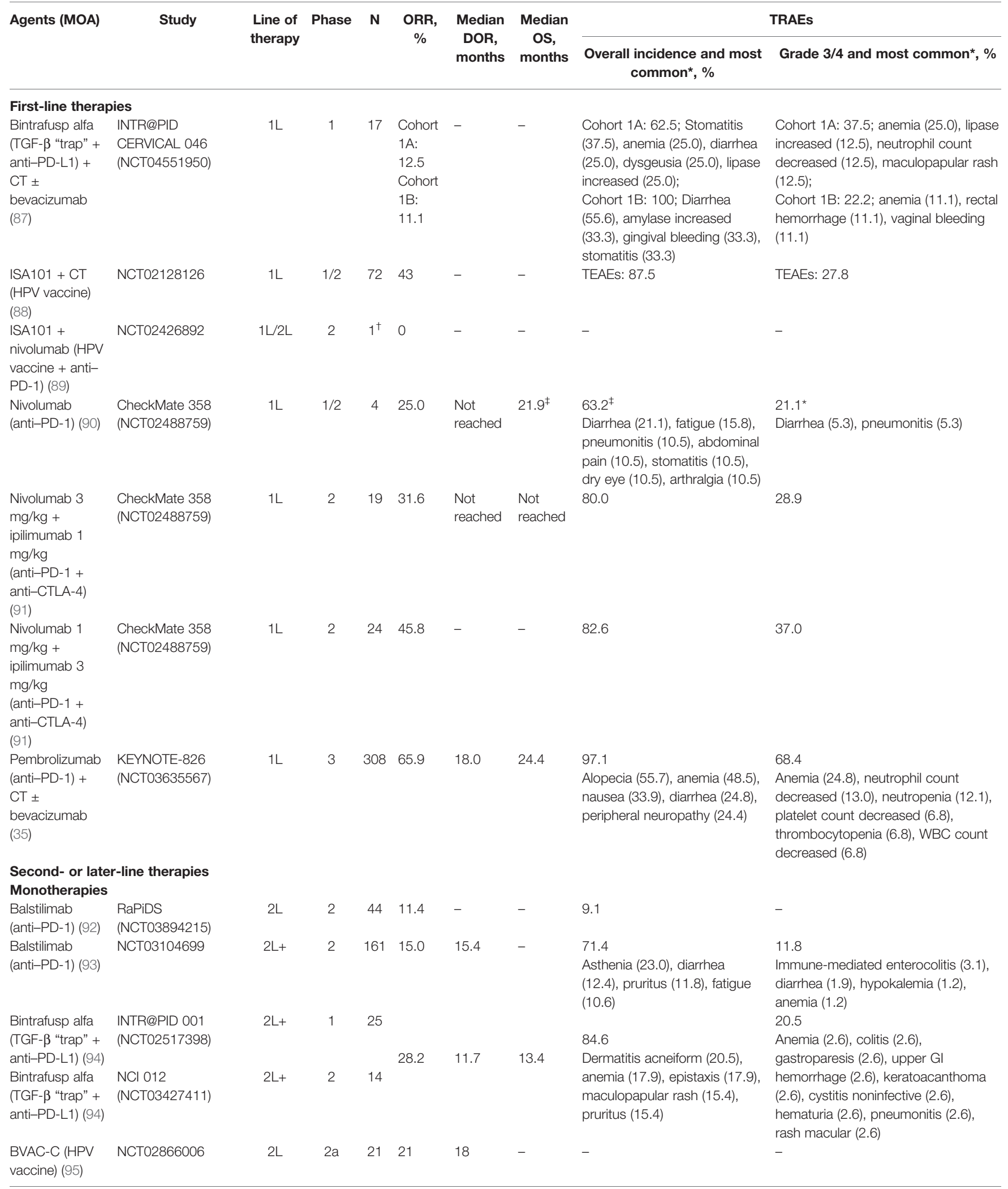


TABLE 1 | Continued

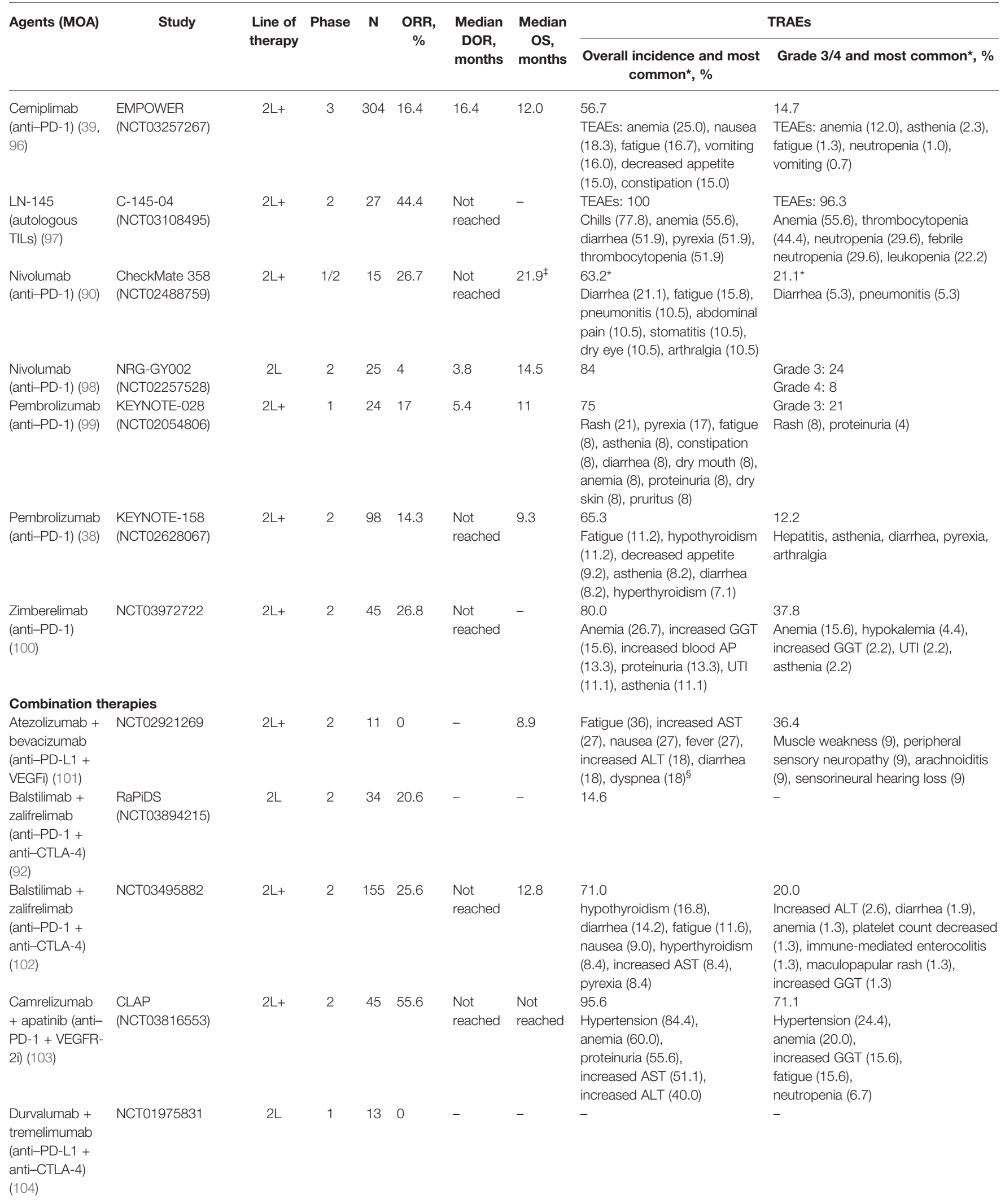


TABLE 1 | Continued

\begin{tabular}{|c|c|c|c|c|c|c|c|c|c|}
\hline \multirow[t]{2}{*}{ Agents (MOA) } & \multirow[t]{2}{*}{ Study } & \multirow{2}{*}{$\begin{array}{l}\text { Line of } \\
\text { therapy }\end{array}$} & \multirow[t]{2}{*}{ Phase } & \multirow[t]{2}{*}{$\mathbf{N}$} & \multirow{2}{*}{$\begin{array}{l}\text { ORR, } \\
\%\end{array}$} & \multirow{2}{*}{$\begin{array}{l}\text { Median } \\
\text { DOR, } \\
\text { months }\end{array}$} & \multirow{2}{*}{$\begin{array}{l}\text { Median } \\
\text { OS, } \\
\text { months }\end{array}$} & \multicolumn{2}{|r|}{ TRAEs } \\
\hline & & & & & & & & $\begin{array}{l}\text { Overall incidence and most } \\
\text { common }{ }^{\star} \%\end{array}$ & Grade $3 / 4$ and most common*, $\%$ \\
\hline $\begin{array}{l}\text { Nivolumab } 3 \\
\mathrm{mg} / \mathrm{kg}+ \\
\text { ipilimumab } 1 \\
\text { mg/kg } \\
\text { (anti-PD-1 + } \\
\text { anti-CTLA-4) } \\
\text { (91) }\end{array}$ & $\begin{array}{l}\text { CheckMate } 358 \\
\text { (NCT02488759) }\end{array}$ & $2 \mathrm{~L}+$ & 2 & 26 & 23.1 & 14.6 & 10.3 & 80.0 & 28.9 \\
\hline $\begin{array}{l}\text { Nivolumab } 1 \\
\mathrm{mg} / \mathrm{kg}+ \\
\text { ipilimumab } 3 \\
\text { mg/kg } \\
\text { (anti-PD-1 + } \\
\text { anti-CTLA-4) } \\
\text { (91) }\end{array}$ & $\begin{array}{l}\text { CheckMate } 358 \\
\text { (NCT02488759) }\end{array}$ & $2 \mathrm{~L}+$ & 2 & 22 & 36.4 & 9.5 & 25.4 & 82.6 & 37.0 \\
\hline $\begin{array}{l}\text { Pembrolizumab } \\
+ \text { GX-188E } \\
\text { (anti-PD-1 + } \\
\text { HPV vaccine) } \\
\text { (105) }\end{array}$ & $\begin{array}{l}\text { KEYNOTE-567 } \\
\text { (NCT03444376) }\end{array}$ & $2 \mathrm{~L}$ & 2 & 52 & 31.1 & - & 16.7 & 32.7 & $\begin{array}{l}3.8 \\
\text { Increased ALT (1.9), increased AST } \\
(1.9)\end{array}$ \\
\hline $\begin{array}{l}\text { Simlukafusp alfa } \\
+ \text { atezolizumab } \\
\text { (FAP-IL-2v + } \\
\text { anti-PD-L1) } \\
\text { (106) }\end{array}$ & NCT03386721 & $2 \mathrm{~L}+$ & 2 & 47 & 27 & 13.3 & - & - & $\begin{array}{l}\text { Grade 3: } 63.8 \\
\text { Grade 4: } 29.8\end{array}$ \\
\hline $\begin{array}{l}\text { Sintilimab + } \\
\text { anlotinib (anti- } \\
\text { PD-1 + TKI) } \\
(107)\end{array}$ & ChiCTR1900023015 & $2 \mathrm{~L}+$ & 2 & 42 & 56.4 & - & - & $\begin{array}{l}\text { Hypothyroidism (33.3), } \\
\text { hypertension (23.8), AST } \\
\text { (21.4), diarrhea (19.0), ALT } \\
\text { (16.7) }\end{array}$ & $\begin{array}{l}\text { Hypertension (4.8), hyponatremia } \\
(4.8) \text {, immune pneumonia (2.4), } \\
\text { immune myocarditis }(2.4)\end{array}$ \\
\hline
\end{tabular}

*Most common preferred term refers to the five most common TRAEs, if reported.

${ }^{t}$ Part of a phase 2 trial enrolling 24 patients with HPV-positive cancers (most of which were SCCHN).

${ }^{\ddagger}$ Includes all patients comprising those who received $1 \mathrm{~L}$ and $2 \mathrm{~L}+$ treatment.

§TRAEs attributed to atezolizumab.

$1 L$, first line; $2 L$, second line; ALT, alanine aminotransferase; AP, alkaline phosphatase; AST, aspartate aminotransferase; CT, chemotherapy; CTLA-4, cytotoxic T-lymphocyte protein 4; $D O R$, duration of response; FAP, fibroblast activation protein- $\alpha$; GGT, $\gamma$ glutamyl transferase; Gl, gastrointestinal; HPV, human papillomavirus; IL-2V, interleukin-2 variant; MOA, mechanism of action; NA, not applicable; ORR, objective response rate; OS, overall survival; PD-1, programmed death 1; PD-L1, programmed death ligand 1; SCCHN, squamous cell carcinoma of the head and neck; TEAE, treatment-emergent adverse event; TGF- $\beta$, transforming growth factor $\beta$; TIL, tumor-infiltrating lymphocyte; TKI, tyrosine kinase inhibitor; TRAE, treatment-related adverse event; UTI, urinary tract infection; VEGFi, vascular endothelial growth factor inhibitor; WBC, white blood cell.

agent chemotherapy $(39,113)$. The improvement in median OS benefit was numerically higher in adenocarcinoma than squamous cell carcinoma when compared with respective chemotherapy results (6.3 vs 2.3 months). Further analysis of these treatments in these histologic subtypes is needed.

The results to date demonstrate that while anti-PD-(L)1 therapies have efficacy as second- or later-line therapy for cervical cancer, not all patients benefit from therapy. Furthermore, resistance to anti-PD-(L)1 therapy remains a concern. Therefore, therapies that address mechanisms of resistance are needed to further improve outcomes in more patients.

\section{Combination Therapies}

Studies of combinations of anti-PD-(L)1 agents with other checkpoint inhibitors (inducible T-cell costimulator, cytotoxic T-lymphocyte protein 4 [CTLA-4], T-cell immunoreceptor with immunoglobulin and immunoreceptor tyrosine-based inhibition motif domains), VEGF inhibitors (bevacizumab, cabozantinib, apatinib), and HPV vaccines (ISA101, GX-188E) reflect the importance of targeting multiple pathways to improve treatment outcomes (Tables 1 and 2). While clinical trials of many of these combinations are ongoing, the most robust evidence exists for combinations of anti-PD-(L) 1 and antiCTLA-4 agents. Different combinations of anti-PD-(L) 1 and anti-CTLA-4 agents have shown ORRs between $20.6 \%$ and $36.4 \%$ in patients who had received prior systemic therapy (Table 1). However, limited efficacy in specific histological types of cervical cancer emphasizes the challenges observed previously with anti-PD-(L)1 monotherapies. Similar to balstilimab monotherapy (93), the combination of balstilimab and zalifrelimab showed lower response rates in patients with cervical adenocarcinoma than those with squamous cell carcinoma ( $8.8 \%$ vs $32.6 \%$ ) (102).

Two small phase 2 studies have evaluated combinations of anti-PD-(L)1 agents and VEGF inhibitors in patients with at least one prior systemic therapy $(101,103)$. Due to small sample sizes and the absence of a comparator arm, results of these 
TABLE 2 | Ongoing clinical trials of immunotherapy agents in cervical cancers.

\begin{tabular}{|c|c|c|c|c|c|c|c|}
\hline Agents (MOA) & Key inclusion criteria & $\begin{array}{l}\text { Combination } \\
\text { partner }\end{array}$ & Study & Phase & $\mathbf{N}$ & Locations & $\begin{array}{l}\text { Primary } \\
\text { completion } \\
\text { date }\end{array}$ \\
\hline \multicolumn{8}{|l|}{ First-line therapies } \\
\hline $\begin{array}{l}\text { Atezolizumab } \\
(\text { anti-PD-L1) (108) }\end{array}$ & $\begin{array}{l}\text { Female; age } \geq 18 \text { y; persistent, recurrent, } \\
\text { or metastatic SCC, AC, or AS; no prior } \\
\text { systemic treatment }\end{array}$ & $\begin{array}{l}\mathrm{CT}+ \\
\text { bevacizumab }\end{array}$ & $\begin{array}{l}\text { BEATCC } \\
\text { (NCT03556839) }\end{array}$ & 3 & 404 & $\begin{array}{l}\text { US, Asia, } \\
\text { and Europe }\end{array}$ & $3 / 2023$ \\
\hline $\begin{array}{l}\text { Pembrolizumab } \\
\text { (anti-PD-1) }\end{array}$ & $\begin{array}{l}\text { Female; age } \geq 18 \text { y; persistent, recurrent, } \\
\text { or metastatic SCC, AC, or AS; no prior } \\
\text { systemic treatment }\end{array}$ & $\begin{array}{l}\mathrm{CT}+ \\
\text { bevacizumab }\end{array}$ & NCT03367871 & 2 & 40 & US & $10 / 2022$ \\
\hline $\begin{array}{l}\text { Pembrolizumab } \\
\text { (anti-PD-1) }\end{array}$ & $\begin{array}{l}\text { Female; age } \geq 18 \text { y; high-risk locally } \\
\text { advanced cancer; SCC, AC, or AS; no } \\
\text { prior systemic treatment }\end{array}$ & CRT & $\begin{array}{l}\text { ENGOT-cX11/GOG } \\
\text { 3047/KEYNOTE-A18 } \\
\text { (NCT04221945) }\end{array}$ & 3 & 980 & Global & $2 / 2024$ \\
\hline Prolgolimab (BCD-100; anti-PD-1) & $\begin{array}{l}\text { Female; age } \geq 18 \text { y; recurrent, or } \\
\text { metastatic cervical cancer; no prior } \\
\text { systemic treatment }\end{array}$ & $\begin{array}{l}\mathrm{CT} \pm \\
\text { bevacizumab }\end{array}$ & $\begin{array}{l}\text { FERMATA } \\
\text { (NCT03912415) }\end{array}$ & 3 & 316 & Global & $12 / 2024$ \\
\hline Prolgolimab (BCD-100; anti-PD-1) & $\begin{array}{l}\text { Female; age } \geq 18 \text { y; persistent, recurrent, } \\
\text { or metastatic SCC, AC, or AS; no prior } \\
\text { systemic treatment }\end{array}$ & $\begin{array}{l}\mathrm{CT}+ \\
\text { bevacizumab }\end{array}$ & $\begin{array}{l}\text { CAESURA } \\
\text { (NCT03912402) }\end{array}$ & 2 & 49 & Russia & $7 / 2020$ \\
\hline Z-100 (Immune-modulator) & $\begin{array}{l}\text { Female; age } \geq 21 \text { y; FIGO stage IIIB; } \\
\text { confirmed SCC; no prior systemic } \\
\text { treatment }\end{array}$ & $\mathrm{RT}$ & NCT03476018 & 3 & 72 & Vietnam & 10/2021 \\
\hline \multicolumn{8}{|l|}{ Second- or later-line therapies } \\
\hline $\begin{array}{l}\text { AK104 } \\
\text { (anti-PD-1 + anti-CTLA-4) }\end{array}$ & $\begin{array}{l}\text { Female; age } \geq 18 \text { y; recurrent or metastatic } \\
\text { SCC or AC; } \geq 1 \text { prior systemic therapy }\end{array}$ & None & NCT04380805 & 2 & 40 & $\begin{array}{l}\text { US and } \\
\text { Australia }\end{array}$ & $8 / 2021$ \\
\hline $\begin{array}{l}\text { Atezolizumab + KY-1044 (anti-PD- } \\
\text { L1 + ICOSi) }\end{array}$ & $\begin{array}{l}\text { Patients; age } \geq 18 \text { y; metastatic cancers; } \\
\text { anti-PD-(L)1 therapy naive and pretreated }\end{array}$ & None & NCT03829501 & $1 / 2$ & $412^{*, \dagger}$ & Global & $5 / 2023$ \\
\hline $\begin{array}{l}\text { Atezolizumab + MG1-E6E7 + Ad- } \\
\text { E6E7 (anti-PD-L1 + anti-HPV } \\
\text { oncolytic virus + HPV vaccine) }\end{array}$ & $\begin{array}{l}\text { Age } \geq 18 \text { y; recurrent or metastatic HPV- } \\
\text { associated cancers; received prior therapy }\end{array}$ & None & $\begin{array}{l}\text { Kingfisher } \\
\text { (NCT03618953) }\end{array}$ & 1 & $75^{\dagger}$ & $\begin{array}{l}\text { US and } \\
\text { Canada }\end{array}$ & $4 / 2021$ \\
\hline $\begin{array}{l}\text { Atezolizumab + tiragolumab (anti- } \\
\text { PD-L1 + anti-TIGIT) }\end{array}$ & $\begin{array}{l}\text { Female; age } \geq 18 \text { y; recurrent or persistent } \\
\text { SCC or AC; } \geq 1 \text { prior systemic CT }\end{array}$ & None & $\begin{array}{l}\text { SKYSCRAPER-04 } \\
\text { (NCT04300647) }\end{array}$ & 2 & $160^{\ddagger}$ & Global & $7 / 2023$ \\
\hline $\begin{array}{l}\text { Atezolizumab } \pm \text { Vigil } \\
\text { (anti-PD-L1 + TGF- } \beta / \text { GM-CSF } \\
\text { vaccine) }\end{array}$ & $\begin{array}{l}\text { Female; age } \geq 18 \text { y; confirmed stage IIIB, } \\
\text { IIIC, or IV or metastatic cancers; recurrent } \\
\text { ovarian disease }\end{array}$ & None & NCT03073525 & 2 & $25^{\dagger}$ & US & $1 / 2021$ \\
\hline $\begin{array}{l}\text { Bintrafusp alfa } \\
\text { (TGF- } \beta \text { "trap" + anti-PD-L1) }\end{array}$ & $\begin{array}{l}\text { Female; age } \geq 18 \text { y; advanced, } \\
\text { unresectable, or metastatic } S C C, A C \text {, or } \\
\text { AS; progression after platinum therapy }\end{array}$ & None & $\begin{array}{l}\text { INTR@PID CERVICAL } \\
017 \\
\text { (NCT04246489) }\end{array}$ & 2 & 146 & Global & $12 / 2021$ \\
\hline $\begin{array}{l}\text { Bintrafusp alfa } \\
\text { (TGF- } \beta \text { “trap" + anti-PD-L1) (109) }\end{array}$ & $\begin{array}{l}\text { Female; age } \geq 18 \text { y; locally advanced or } \\
\text { metastatic HPV-associated cancers; } \geq 1 \\
\text { prior anticancer therapy }\end{array}$ & None & $\begin{array}{l}\mathrm{NCl} 012 \\
(\mathrm{NCT03427411)}\end{array}$ & 2 & $57^{\dagger}$ & US & $12 / 2022$ \\
\hline $\begin{array}{l}\text { Dostarlimab + niraparib (anti-PD-1 } \\
\text { + PARPi) }\end{array}$ & $\begin{array}{l}\text { Female; age } \geq 18 \text { y; recurrent or } \\
\text { progressive cervical cancer; } \geq 1 \text { prior } \\
\text { systemic therapy }\end{array}$ & None & $\begin{array}{l}\text { STAR } \\
\text { (NCT04068753) }\end{array}$ & 2 & 66 & US & $7 / 2023$ \\
\hline $\begin{array}{l}\text { Durvalumab + MEDI-0457 (anti-PD- } \\
\text { L1 + HPV vaccine) }\end{array}$ & $\begin{array}{l}\text { Age } \geq 18 \text { y; recurrent or metastatic HPV- } \\
\text { associated cancers; refractory or relapsed } \\
\text { after standard therapy }\end{array}$ & None & NCT03439085 & 2 & $77^{\dagger}$ & US & $12 / 2021$ \\
\hline $\begin{array}{l}\text { Durvalumab + tremelimumab } \\
\text { (anti-PD-L1 + anti-CTLA-4) }\end{array}$ & $\begin{array}{l}\text { Female; age } \geq 18 \text { y; recurrent or metastatic } \\
\text { gynecologic cancer; progression on } \\
\text { platinum-based CT }\end{array}$ & $\mathrm{RT}$ & NCT03277482 & 1 & $32^{\dagger}$ & US & $1 / 2022$ \\
\hline Genolimzumab (GB-226; anti-PD-1) & $\begin{array}{l}\text { Female; age } \geq 18 \text { y; recurrent, or } \\
\text { metastatic cervical cancer; progression } \\
\text { after platinum therapy }\end{array}$ & None & NCT03808857 & 2 & $80^{\ddagger}$ & China & $12 / 2020$ \\
\hline $\begin{array}{l}\text { Gemogenovatucel-T + durvalumab } \\
\text { (TGF- } \beta \text { /GM-CSF vaccine + anti-PD- } \\
\text { L1) }\end{array}$ & $\begin{array}{l}\text { Female; age } \geq 18 \text { y; locally advanced or } \\
\text { metastatic cancers; treatment naive or } \\
\text { resistant to anti-PD-(L)1 therapy }\end{array}$ & None & NCT02725489 & 2 & $13^{\star, \dagger}$ & US & $12 / 2019$ \\
\hline $\begin{array}{l}\text { NP137 + pembrolizumab (anti- } \\
\text { Netrin-1 + anti-PD-1) }\end{array}$ & $\begin{array}{l}\text { Female; age } \geq 18 \text { y; recurrent cervical } \\
\text { cancer; } \geq 1 \text { prior } \mathrm{CT} \text { regimen }\end{array}$ & CT & $\begin{array}{l}\text { GYNET } \\
(\text { NCT04652076) }\end{array}$ & $1 / 2$ & 240 & France & $10 / 2021$ \\
\hline $\begin{array}{l}\text { Pembrolizumab + cabozantinib } \\
\text { (anti-PD-1 + VEGFRi) }\end{array}$ & $\begin{array}{l}\text { Female; age } \geq 18 \text { y; recurrent or persistent } \\
\text { SCC, AC, or AS; prior systemic CT }\end{array}$ & None & NCT04230954 & 2 & $39^{\ddagger}$ & US & $6 / 2021$ \\
\hline $\begin{array}{l}\text { Pembrolizumab + olaparib (anti-PD- } \\
1 \text { + PARPi) (110) }\end{array}$ & $\begin{array}{l}\text { Female; age } \geq 18 \text { y; recurrent cervical } \\
\text { cancer; } \geq 1 \text { prior } \mathrm{CT} \text { regimen }\end{array}$ & None & NCT04483544 & 2 & 48 & US & $11 / 2030$ \\
\hline $\begin{array}{l}\text { Tislelizumab + ociperlimab (anti-PD- } \\
1+\text { anti-TIGIT) (111) }\end{array}$ & $\begin{array}{l}\text { Female; age } \geq 18 \text { y; } \mathrm{SCC}, \mathrm{AC} \text {, or } \mathrm{AS} ; \geq 1 \\
\text { prior } \mathrm{CT}\end{array}$ & None & $\begin{array}{l}\text { AdvanTIG-202 } \\
\text { (NCT04693234) }\end{array}$ & 2 & 167 & $\begin{array}{l}\text { China, } \\
\text { South }\end{array}$ & 3/2022 \\
\hline
\end{tabular}


TABLE 2 | Continued

\begin{tabular}{|c|c|c|c|c|c|c|c|}
\hline Agents (MOA) & Key inclusion criteria & $\begin{array}{l}\text { Combination } \\
\text { partner }\end{array}$ & Study & Phase & $\mathbf{N}$ & Locations & $\begin{array}{c}\text { Primary } \\
\text { completion } \\
\text { date }\end{array}$ \\
\hline $\begin{array}{l}\text { Tucatinib + trastuzumab (tyrosine } \\
\text { kinase inhibitor + HER2 inhibitor) }\end{array}$ & $\begin{array}{l}\text { Female; age } \geq 18 \text { y; metastatic cancer; } \geq 1 \\
\text { prior systemic therapy }\end{array}$ & None & $\begin{array}{l}\text { SGNTUC-019 } \\
\text { (NCT04579380) }\end{array}$ & 2 & $270^{\dagger}$ & $\begin{array}{l}\text { Korea, and } \\
\text { Taiwan } \\
\text { US }\end{array}$ & $1 / 2023$ \\
\hline \multicolumn{8}{|c|}{$\begin{array}{l}\text { *Some patients enrolled in this study will have received one or two prior lines of therapy for recurrent/metastatic cervical cancer. } \\
{ }^{+} \text {Not all patients enrolled in this study will have cervical cancer. } \\
{ }^{\ddagger} \text { Patients must have PD-L1-positive tumors. } \\
\text { AC, adenocarcinoma; AS, adenosquamous; CRT, chemoradiotherapy; CT, chemotherapy; CTLA-4, cytotoxic T-lymphocyte protein 4; FIGO, Federation Internationale de Gynecolgie et } \\
d^{\prime} \text { Obstetrique; GM-CSF, granulocyte-macrophage colony-stimulating factor; HER2, human epidermal growth factor receptor 2; HPV, human papilloma virus; ICOSI, inducible T-cell } \\
\text { costimulator inhibitor; MOA, mechanism of action; PARPi, poly (adenosine diphosphate-ribose) polymerase inhibitor; PD-1, programmed death 1; PD-L1, programmed death ligand 1; RT, } \\
\text { radiotherapy; SCC, squamous cell carcinoma; TGF- } \beta \text {, transforming growth factor } \beta \text {; TIGIT, T-cell immunoreceptor with immunoglobulin and immunoreceptor tyrosine-based inhibition } \\
\text { motif domains; VEGFRi, vascular endothelial growth factor receptor inhibitor. }\end{array}$} \\
\hline
\end{tabular}

studies need to be validated in larger trials. Several studies have demonstrated that poly (adenosine diphosphate-ribose) polymerase (PARP) inhibitors have antiangiogenic activity, the ability to promote infiltration of $\mathrm{CD} 8+\mathrm{T}$ cells, and a role in maintaining genomic stability (116-120). In addition, PARP inhibitors can decrease TGF- $\beta$ expression, leading to inhibition of EMT and fibrosis $(121,122)$. Based on these observations, two phase 2 studies are examining combinations of anti-PD-(L)1 agents with PARP inhibitors (niraparib, olaparib) (Table 2). One phase 2 study has shown promising activity with simlukafusp alfa, a bifunctional antibody, in combination with anti-PD-L1; however, considerable toxicity seems to be associated with this combination, with $63.4 \%$ and $29.8 \%$ of patients reporting grade 3 and 4 treatment-related AEs, respectively (106).

\section{Dual Targeting Therapy to Inhibit TGF- $\beta$ and PD-L1}

As previously described, in addition to PD-L1, TGF- $\beta$ may play a significant and related but nonredundant role in the underlying pathophysiology of cervical cancer, providing a rationale for inhibiting both pathways simultaneously. Bintrafusp alfa is a first-in-class bifunctional fusion protein composed of the extracellular domain of the human TGF- $\beta$ receptor II (TGF$\beta$ RII or TGF- $\beta$ "trap") fused via a flexible linker to the Cterminus of each heavy chain of an IgG1 antibody blocking PDL1 (anti-PD-L1) (123-125). Bintrafusp alfa is designed to block TGF- $\beta$ signaling by "trapping" all TGF- $\beta$ isoforms $(\beta 1, \beta 2$, and $\beta 3$ ), and this "trap" function is physically linked to PD-L1 blockade in the TME $(123,124)$. Bintrafusp alfa targets tumors via simultaneous blocking of the immunosuppressive TGF- $\beta$ and PD-L1 pathways within the TME and is the only agent currently under investigation in cervical cancer that targets both pathways. Studies in murine squamous cell carcinoma models showed that anti-PD-(L)1 therapy rarely led to complete regression, but that adding anti-TGF- $\beta$ synergistically enhanced antitumor responses. The synergy was partly driven by anti-TGF- $\beta$-mediated suppression of anti-PD-(L)1 resistance and by EMT attenuation and immunosurveillance stimulation $(109,126)$. In mouse models, the combination of bintrafusp alfa and chemotherapy significantly reduced tumor growth relative to either treatment alone (123). In these models, bintrafusp alfa also significantly increased $\mathrm{T}$ cells, natural killer cells, macrophages, and dendritic cells and decreased neutrophils and myeloid-derived suppressor cells in tumor tissue compared with anti-PD-L1 or a TGF- $\beta$ "trap" control (123). Furthermore, as noted above, upregulation of TGF- $\beta$ signaling and expression in the TME after radiation and chemotherapy treatments may also attenuate the efficacy of, or even promote resistance to, these anticancer therapies (42-44).

To date, bintrafusp alfa is the only agent targeting both TGF- $\beta$ and PD-L1 to demonstrate efficacy in pretreated recurrent or metastatic cervical cancer. Two clinical studies, INTR@PID 001 (NCT02517398) and NCI 012 (NCT03427411), have evaluated bintrafusp alfa in cohorts of patients with pretreated recurrent or metastatic cervical cancer. In a cohort of 25 heavily pretreated patients with recurrent or metastatic cervical cancer from the phase 1 INTR@PID 001 clinical trial who had disease progression with prior platinum-containing chemotherapy, the ORR was $24.0 \%$ per investigator assessment. One patient had a delayed partial response after initial disease progression (127). In a post hoc pooled analysis of patients with recurrent or metastatic cervical cancer from the INTR@PID 001 study and a phase 2 single-center study (NCI 012), the ORR was $28.2 \%$ (95\% CI, $15.0 \%-44.9 \%$ ) (94). Responses to bintrafusp alfa were longlasting, with a median DOR of 11.7 months (range, 1.4-41.2 months), and were observed irrespective of tumor histology or prior bevacizumab treatment. In contrast, anti-PD-(L)1 therapies tend to be less effective in patients with adenocarcinoma $(93,102$, $115)$ and those who have been exposed to bevacizumab $(37,102)$. Additionally, the safety profile of bintrafusp alfa in patients with heavily pretreated recurrent or metastatic cervical cancer who had disease progression with platinum-containing chemotherapy was consistent with that of anti-PD-(L) 1 therapies, except for treatment-related AEs known to be associated with TGF- $\beta$ inhibitors (e.g., localized skin lesions including keratoacanthomas) $(94,109)$.

Bintrafusp alfa has also shown potential to treat other rare HPVrelated tumors, such as anal, rectal, and vaginal cancer in INTR@ PID 001 and NCI 012 trials; the overall ORR was $28.0 \%$ in patients with HPV-associated malignancies $(109,128)$. Anti-PD-1 therapy with nivolumab has also shown a promising response rate of $20.0 \%$ in patients with vaginal or vulvar cancers in CheckMate 358 (90). 
These data indicate the potential benefit of bintrafusp alfa monotherapy in the postplatinum recurrent or metastatic setting. This is being explored further in a large phase 2 trial (NCT04246489). In addition, a phase 1 study is investigating the safety of bintrafusp alfa in combination with other anticancer therapies in patients with locally advanced or advanced cervical cancer (NCT04551950). Preliminary results from this study showed no new safety signals, and two partial responses among six patients who had a tumor assessment in patients with advanced cervical cancer (87).

\section{CONCLUSIONS}

Treatment options are limited in patients with persistent, recurrent, or metastatic cervical cancer. Although several immunotherapies, including anti-PD-(L)1 agents, have shown clinical activity in persistent, recurrent, or metastatic cervical cancer, response rates are relatively low and vary based on PD-L1 expression and tumor histology. Agents that target immunosuppressive mechanisms within the TME associated with HPV infection, such as bintrafusp alfa, which simultaneously targets the TGF- $\beta$ and PDL1 pathways, are under investigation. Promising results were observed with bintrafusp alfa in phase $1 / 2$ trials in patients with persistent, recurrent, or metastatic cervical cancer.

\section{FUTURE PERSPECTIVES}

Although bintrafusp alfa has shown promising results in patients with persistent, recurrent, or metastatic cervical cancer in phases 1-3, strategies for patient selection, including assessment of PDL1 status, tumor histology, and TGF- $\beta$ signature profile, are necessary. In the phase 1 INTR@PID 001 clinical trial, PD-L1 expression was detected by immunohistochemistry in a fraction of patients; a threshold of $1 \%$ was used to characterize tumors as PD-L1 positive $(\geq 1 \%)$ or negative $(<1 \%)$ (109). Treatment

\section{REFERENCES}

1. Sung H, Ferlay J, Siegel RL, Laversanne M, Soerjomataram I, Jemal A, et al. Global Cancer Statistics 2020: GLOBOCAN Estimates of Incidence and Mortality Worldwide for 36 Cancers in 185 Countries. CA Cancer J Clin (2021) 71(3):209-49. doi: 10.3322/caac.21660

2. Arbyn M, Weiderpass E, Bruni L, de Sanjosé S, Saraiya M, Ferlay J, et al. Estimates of Incidence and Mortality of Cervical Cancer in 2018: A Worldwide Analysis. Lancet Glob Health (2020) 8(2):e191-203. doi: 10.1016/S2214-109X(19)30482-6

3. Marth C, Landoni F, Mahner S, McCormack M, Gonzalez-Martin A, Colombo N, et al. Cervical Cancer: ESMO Clinical Practice Guidelines for Diagnosis, Treatment and Follow-Up. Ann Oncol (2017) 28(suppl 4):iv7283. doi: $10.1093 /$ annonc/mdx 220

4. American Cancer Society. What Is Cervical Cancer? (2020). Available at: https://www.cancer.org/cancer/cervical-cancer/about/what-is-cervicalcancer.html (Accessed September 30, 2021).

5. National Cancer Institute. Surveillance, Epidemiology, and End Results Program . Available at: https://seer.cancer.gov/ (Accessed September 30, 2021).

6. Bielska-Lasota M, Rossi S, Krzyżak M, Haelens A, Domenic A, De Angelis R, et al. Group: Reasons for Low Cervical Cancer Survival in New Accession responses occurred irrespective of PD-L1 status. In a pooled analysis of patients from the INTR@PID 001 and NCI 012 trials, responses to bintrafusp alfa were observed irrespective of tumor histology (94), although a systematic approach in a larger trial might shed additional insights.

The TGF- $\beta$ signature profile can identify patients with gynecologic cancers who are likely to benefit from immune checkpoint inhibitors (129). This approach may also be used to select patients who may respond to bintrafusp alfa. Another approach can be to investigate if tumor mutational burden (TMB) can be used as a biomarker for this bifunctional agent; TMB has been demonstrated to be a useful biomarker for immune checkpoint inhibitor therapy across cancer types (130). Future trials are needed to clarify the role of biomarkers to identify the patient population that would benefit from bintrafusp alfa treatment.

\section{AUTHOR CONTRIBUTIONS}

All authors contributed to the conception and design of the manuscript and revised, read, and approved the submitted version.

\section{FUNDING}

This work was funded by Merck (CrossRef Funder ID: 10.13039/ 100009945) and was previously part of an alliance between Merck and GlaxoSmithKline.

\section{ACKNOWLEDGMENTS}

Medical writing support was provided by Kakoli Parai, ClinicalThinking, Inc, which was also funded by Merck and GlaxoSmithKline in accordance with Good Publication Practice (GPP3) guidelines (http://www.ismpp.org/gpp3).

European Union Countries: A EUROCARE-5 Study. Arch Gynecol Obstet (2020) 301(2):591-602. doi: 10.1007/s00404-019-05412-5

7. Yamagami W, Nagase S, Takahashi F, Ino K, Hachisuga T, Aoki D, et al. Clinical Statistics of Gynecologic Cancers in Japan. J Gynecol Oncol (2017) 28(2):e32. doi: 10.3802/jgo.2017.28.e32

8. Watanabe T, Mikami M, Katabuchi H, Kato S, Kaneuchi M, Takahashi M, et al. Quality Indicators for Cervical Cancer Care in Japan. J Gynecol Oncol (2018) 29(6):e83. doi: 10.3802/jgo.2018.29.e83

9. Canfell K, Kim JJ, Brisson M, Keane A, Simms KT, Caruana M, et al. Mortality Impact of Achieving WHO Cervical Cancer Elimination Targets: A Comparative Modelling Analysis in 78 Low-Income and Lower-Middle-Income Countries. Lancet (2020) 395(10224):591-603. doi: 10.1016/S0140-6736(20)30157-4

10. World Health Organization. Human Papillomavirus (HPV) and Cervical Cancer. (2020). Available at: https://www.who.int/news-room/fact-sheets/detail/humanpapillomavirus-(hpv)-and-cervical-cancer (Accessed September 30, 2021).

11. American Cancer Society. Signs and Symptoms of Cervical Cancer. (2020). Available at: https://www.cancer.org/cancer/cervical-cancer/detectiondiagnosis-staging/signs-symptoms.html (Accessed September 30, 2021).

12. Friedlander M, Grogan M. Guidelines for the Treatment of Recurrent and Metastatic Cervical Cancer. Oncologist (2002) 7(4):342-7. doi: 10.1634/ theoncologist.2002-0342 
13. World Health Organization. Comprehensive Cervical Cancer Control: A Guide to Essential Practice. 2nd ed. Geneva, Switzerland: World Health Organization (2014).

14. Maduro JH, Pras E, Willemse PH, de Vries EG. Acute and Long-Term Toxicity Following Radiotherapy Alone or in Combination With Chemotherapy for Locally Advanced Cervical Cancer. Cancer Treat Rev (2003) 29(6):471-88. doi: 10.1016/s0305-7372(03)00117-8

15. Gondi V, Bentzen SM, Sklenar KL, Dunn EF, Petereit DG, Tannehill SP, et al. Severe Late Toxicities Following Concomitant Chemoradiotherapy Compared to Radiotherapy Alone in Cervical Cancer: An Inter-Era Analysis. Int J Radiat Oncol Biol Phys (2012) 84(4):973-82. doi: 10.1016/ j.ijrobp.2012.01.064

16. Sasidharan A, Mahantshetty UM, Gurram L, Chopra S, Engineer R, Maheshwari A, et al. Patterns of First Relapse and Outcome in Patients With Locally Advanced Cervical Cancer After Radiochemotherapy: A Single Institutional Experience. Indian J Gynecol Oncol (2019) 18(1):4. doi: 10.1007/s40944-019-0345-7

17. Boussios S, Seraj E, Zarkavelis G, Petrakis D, Kollas A, Kafantari A, et al. Management of Patients With Recurrent/Advanced Cervical Cancer Beyond First Line Platinum Regimens: Where do We Stand? A Literature Review. Crit Rev Oncol Hematol (2016) 108:164-74. doi: 10.1016/ j.critrevonc.2016.11.006

18. Pfaendler KS, Wenzel L, Mechanic MB, Penner KR. Cervical Cancer Survivorship: Long-Term Quality of Life and Social Support. Clin Ther (2015) 37(1):39-48. doi: 10.1016/j.clinthera.2014.11.013

19. Le Borgne G, Mercier M, Woronoff AS, Guizard AV, Abeilard E, CaravatiJouvenceaux A, et al. Quality of Life in Long-Term Cervical Cancer Survivors: A Population-Based Study. Gynecol Oncol (2013) 129(1):222-8. doi: 10.1016/j.ygyno.2012.12.033

20. Walboomers JM, Jacobs MV, Manos MM, Bosch FX, Kummer JA, Shah KV, et al. Human Papillomavirus Is a Necessary Cause of Invasive Cervical Cancer Worldwide. J Pathol (1999) 189(1):12-9. doi: 10.1002/(SICI)10969896(199909)189:1<12::AID-PATH431>3.0.CO;2-F

21. de Martel C, Georges D, Bray F, Ferlay J, Clifford GM. Global Burden of Cancer Attributable to Infections in 2018: A Worldwide Incidence Analysis. Lancet Glob Health (2020) 8(2):e180-90. doi: 10.1016/S2214-109X(19) 30488-7

22. Clifford G, Franceschi S, Diaz M, Munoz N, Villa LL. Chapter 3: HPV TypeDistribution in Women With and Without Cervical Neoplastic Diseases. Vaccine (2006) 24 Suppl 3:S3/26-34. doi: 10.1016/j.vaccine.2006.05.026

23. International Agency for Research on Cancer. Cervix Cancer Screening. Lyon, France: IARC Press (2005).

24. Tomaic V. Functional Roles of E6 and E7 Oncoproteins in HPV-Induced Malignancies at Diverse Anatomical Sites. Cancers (Basel) (2016) 8(10). doi: $10.3390 /$ cancers 8100095

25. Burd EM, Dean CL. Human Papillomavirus. Microbiol Spectr (2016) 4(4). doi: 10.1128/microbiolspec.DMIH2-0001-2015

26. National Comprehensive Cancer Network. NCCN Clinical Practice Guidelines in Oncology Cervical Cancer. V1. Plymouth Meeting, Pennsylvania: National Comprehensive Cancer Network (2022).

27. Avastin (bevacizumab). Prescribing Information (2021). Genentech, Inc. Available at: https://www.gene.com/download/pdf/avastin_prescribing.pdf (Accessed September 30, 2021).

28. Tewari KS, Sill MW, Long HJ, Penson RT, Huang H, Ramondetta LM, et al. Improved Survival With Bevacizumab in Advanced Cervical Cancer. $N$ Engl J Med (2014) 370(8):734-43. doi: 10.1056/NEJMoa1309748

29. Tewari KS, Sill MW, Penson RT, Huang H, Ramondetta LM, Landrum LM, et al. Bevacizumab for Advanced Cervical Cancer: Final Overall Survival and Adverse Event Analysis of a Randomised, Controlled, Open-Label, Phase 3 Trial (Gynecologic Oncology Group 240). Lancet (2017) 390(10103):165463. doi: 10.1016/S0140-6736(17)31607-0

30. Avastin (bevacizumab). Summary of Product Characteristics. Roche Pharma AG. Available at: https://www.ema.europa.eu/en/documents/productinformation/avastin-epar-product-information_en.pdf (Accessed September 30, 2021).

31. Kitagawa R, Katsumata N, Shibata T, Kamura T, Kasamatsu T, Nakanishi T, et al. Paclitaxel Plus Carboplatin Versus Paclitaxel Plus Cisplatin in Metastatic or Recurrent Cervical Cancer: The Open-Label Randomized
Phase III Trial JCOG0505. J Clin Oncol (2015) 33(19):2129-35. doi: 10.1200/JCO.2014.58.4391

32. Redondo A, Colombo N, McCormack M, Dreosti L, Nogueira-Rodrigues A, Scambia G, et al. Primary Results From CECILIA, A Global Single-Arm Phase II Study Evaluating Bevacizumab, Carboplatin and Paclitaxel for Advanced Cervical Cancer. Gynecol Oncol (2020) 159(1):142-9. doi: 10.1016/j.ygyno.2020.07.026

33. US Food and Drug Administration. FDA Approves Pembrolizumab Combination for the First-Line Treatment of Cervical Cancer. (2021). Available at: https://www.fda.gov/drugs/resources-information-approveddrugs/fda-approves-pembrolizumab-combination-first-line-treatmentcervical-cancer (Accessed November 11, 2021).

34. Keytruda (pembrolizumab). Prescribing Information. (2021). Merck \& Co. Available at: https://www.merck.com/product/usa/pi_circulars/k/keytruda/ keytruda_pi.pdf (Accessed November 11, 2021).

35. Colombo N, Dubot C, Lorusso D, Caceres MV, Hasegawa K, ShapiraFrommer R, et al. Pembrolizumab for Persistent, Recurrent, or Metastatic Cervical Cancer. N Engl J Med (2021) 385(20):1856-67. doi: 10.1056/ NEJMoa2112435

36. US Food and Drug Administration. FDA Approves Pembrolizumab for Advanced Cervical Cancer With Disease Progression During or After Chemotherapy. (2020). Available at: https://www.fda.gov/drugs/resourcesinformation-approved-drugs/fda-approves-pembrolizumab-advancedcervical-cancer-disease-progression-during-or-after-chemotherapy (Accessed September 30, 2021).

37. Chung HC, Ros W, Delord JP, Perets R, Italiano A, Shapira-Frommer R, et al. Efficacy and Safety of Pembrolizumab in Previously Treated Advanced Cervical Cancer: Results From the Phase II KEYNOTE-158 Study. J Clin Oncol (2019) 37(17):1470-8. doi: 10.1200/JCO.18.01265

38. Chung H, Delord J-P, Perets R, Italiano A, Shapira-Frommer R, Manzuk L, et al. Pembrolizumab Treatment of Advanced Cervical Cancer: Updated Results From the Phase II KEYNOTE-158 Study. Gynecol Oncol (2021) 162 (suppl 1):Abstract 41. doi: 10.1016/S0090-8258(21)00696-X

39. Tewari K, Monk BJ, Vergote I, Miller A, de Melo AC, Kim HS, et al EMPOWER-Cervical 1/GOG-3016/ENGOT-Cx9: Interim Analysis of Phase III Trial of Cemiplimab vs Investigator's Choice Chemotherapy in Recurrent/Metastatic Cervical Carcinoma. Ann Oncol (2021) 32(7): Abstract VP4-2021. doi: 10.1016/j.annonc.2021.04.009

40. Rodriguez AC, Schiffman M, Herrero R, Wacholder S, Hildesheim A, Castle PE, et al. And Proyecto Epidemiologico Guanacaste Group: Rapid Clearance of Human Papillomavirus and Implications for Clinical Focus on Persistent Infections. J Natl Cancer Inst (2008) 100(7):513-7. doi: 10.1093/jnci/djn044

41. Centers for Disease Control and Prevention. Basic Information About HPV and Cancer. (2020). Available at: https://www.cdc.gov/cancer/hpv/basic info/index.htm (Accessed September 30, 2021).

42. Torres-Poveda K, Bahena-Roman M, Madrid-Gonzalez C, Burguete-Garcia AI, Bermudez-Morales VH, Peralta-Zaragoza O, et al. Role of IL-10 and TGF- $\beta 1$ in Local Immunosuppression in HPV-Associated Cervical Neoplasia. World J Clin Oncol (2014) 5(4):753-63. doi: 10.5306/ wjco.v5.i4.753

43. Alcocer-Gonzalez JM, Berumen J, Tamez-Guerra R, Bermudez-Morales V, Peralta-Zaragoza O, Hernandez-Pando R, et al. In Vivo Expression of Immunosuppressive Cytokines in Human Papillomavirus-Transformed Cervical Cancer Cells. Viral Immunol (2006) 19(3):481-91. doi: 10.1089/ vim.2006.19.481

44. Xu Q, Wang S, Xi L, Wu S, Chen G, Zhao Y, et al. Effects of Human Papillomavirus Type 16 E7 Protein on the Growth of Cervical Carcinoma Cells and Immuno-Escape Through the TGF-Betal Signaling Pathway. Gynecol Oncol (2006) 101(1):132-9. doi: 10.1016/j.ygyno.2005.09.051

45. Qin Y, Ekmekcioglu S, Forget MA, Szekvolgyi L, Hwu P, Grimm EA, et al. Cervical Cancer Neoantigen Landscape and Immune Activity Is Associated With Human Papillomavirus Master Regulators. Front Immunol (2017) 8:689. doi: $10.3389 /$ fimmu.2017.00689

46. Yang W, Lu YP, Yang YZ, Kang JR, Jin YD, Wang HW. Expressions of Programmed Death (PD)-1 and PD-1 Ligand (PD-L1) in Cervical Intraepithelial Neoplasia and Cervical Squamous Cell Carcinomas Are of Prognostic Value and Associated With Human Papillomavirus Status. J Obstet Gynaecol Res (2017) 43(10):1602-12. doi: 10.1111/jog.13411 
47. Peralta-Zaragoza O, Bermúdez-Morales V, Gutiérrez-Xicotencatl L, Alcocer-González J, Recillas-Targa F, Madrid-Marina V. E6 and E7 Oncoproteins From Huma Papillomavirus Type 16 Induce Activation of Human Transforming Growth Factor Beta1 Promoter Throughout Sp1 Recognition Sequence. Viral Immunol (2006) 19(3):468-80. doi: 10.1089/ vim.2006.19.468

48. Chen J, Deng Y, Ao L, Song Y, Xu Y, Wang CC, et al. The High-Risk HPV Oncogene E7 Upregulates miR-182 Expression Through the TGF- $\beta / S$ mad Pathway in Cervical Cancer. Cancer Lett (2019) 460:75-85. doi: 10.1016/ j.canlet.2019.06.015

49. DeFilippis RA, Goodwin EC, Wu L, DiMaio D. Endogenous Human Papillomavirus E6 and E7 Proteins Differentially Regulate Proliferation, Senescence, and Apoptosis in HeLa Cervical Carcinoma Cells. J Virol (2003) 77(2):1551-63. doi: 10.1128/jvi.77.2.1551-1563.2003

50. Colak S, Ten Dijke P. Targeting TGF- $\beta$ Signaling in Cancer. Trends Cancer (2017) 3(1):56-71. doi: 10.1016/j.trecan.2016.11.008

51. Iancu IV, Botezatu A, Goia-Rusanu CD, Stanescu A, Huica I, Nistor E, et al. TGF-Beta Signalling Pathway Factors in HPV-Induced Cervical Lesions. Roum Arch Microbiol Immunol (2010) 69(3):113-8.

52. Torng PL, Chan WY, Lin CT, Huang SC. Decreased Expression of Human Papillomavirus E2 Protein and Transforming Growth Factor-Betal in Human Cervical Neoplasia as an Early Marker in Carcinogenesis. J Surg Oncol (2003) 84(1):17-23. doi: 10.1002/jso.10273

53. Hao Y, Baker D, Ten Dijke P. TGF- $\beta$-Mediated Epithelial-Mesenchymal Transition and Cancer Metastasis. Int J Mol Sci (2019) 20(11). doi: 10.3390/ ijms 20112767

54. Lee MY, Shen MR. Epithelial-Mesenchymal Transition in Cervical Carcinoma. Am J Transl Res (2012) 4(1):1-13.

55. Yi JY, Hur KC, Lee E, Jin YJ, Arteaga CL, Son YS. TGFbeta1-Mediated Epithelial to Mesenchymal Transition Is Accompanied by Invasion in the SiHa Cell Line. Eur J Cell Biol (2002) 81(8):457-68. doi: 10.1078/0171-933500265

56. Fan Q, Qiu MT, Zhu Z, Zhou JH, Chen L, Zhou Y, et al. Twist Induces Epithelial-Mesenchymal Transition in Cervical Carcinogenesis by Regulating the TGF- $\beta /$ Smad3 Signaling Pathway. Oncol Rep (2015) 34 (4):1787-94. doi: 10.3892/or.2015.4143

57. Tomao F, Papa A, Rossi L, Zaccarelli E, Caruso D, Zoratto F, et al. Angiogenesis and Antiangiogenic Agents in Cervical Cancer. Onco Targets Ther (2014) 7:2237-48. doi: 10.2147/OTT.S68286

58. Principe DR, Doll JA, Bauer J, Jung B, Munshi HG, Bartholin L, et al. TGF- $\beta$ : Duality of Function Between Tumor Prevention and Carcinogenesis. J Natl Cancer Inst (2014) 106(2):djt369. doi: 10.1093/jnci/djt369

59. Biernacka A, Dobaczewski M, Frangogiannis NG. TGF- $\beta$ Signaling in Fibrosis. Growth Factors (2011) 29(5):196-202. doi: 10.3109/08977194.2011.595714

60. Wen S, Niu Y, Yeh S, Chang C. BM-MSCs Promote Prostate Cancer Progression via the Conversion of Normal Fibroblasts to CancerAssociated Fibroblasts. Int J Oncol (2015) 47(2):719-27. doi: 10.3892/ ijo.2015.3060

61. Sahai E, Astsaturov I, Cukierman E, DeNardo DG, Egeblad M, Evans RM, et al. A Framework for Advancing Our Understanding of Cancer-Associated Fibroblasts. Nat Rev Cancer (2020) 20(3):174-86. doi: 10.1038/s41568-0190238-1

62. Torres S, Garcia-Palmero I, Herrera M, Bartolomé RA, Peña C, FernandezAceñero MJ, et al. LOXL2 Is Highly Expressed in Cancer-Associated Fibroblasts and Associates to Poor Colon Cancer Survival. Clin Cancer Res (2015) 21(21):4892-902. doi: 10.1158/1078-0432.CCR-14-3096

63. Wong CC, Tse AP, Huang YP, Zhu YT, Chiu DK, Lai RK, et al. Lysyl Oxidase-Like 2 Is Critical to Tumor Microenvironment and Metastatic Niche Formation in Hepatocellular Carcinoma. Hepatology (2014) 60 (5):1645-58. doi: 10.1002/hep.27320

64. Malanchi I, Santamaria-Martínez A, Susanto E, Peng H, Lehr HA, Delaloye JF, et al. Interactions Between Cancer Stem Cells and Their Niche Govern Metastatic Colonization. Nature (2011) 481(7379):85-9. doi: 10.1038/ nature10694

65. Sakai S, Sato K, Tabata Y, Kishi K. Local Release of Pioglitazone (a Peroxisome Proliferator-Activated Receptor $\gamma$ Agonist) Accelerates Proliferation and Remodeling Phases of Wound Healing. Wound Repair Regener (2016) 24(1):57-64. doi: 10.1111/wrr.12376
66. Chu TY, Yang JT, Huang TH, Liu HW. Crosstalk With Cancer-Associated Fibroblasts Increases the Growth and Radiation Survival of Cervical Cancer Cells. Radiat Res (2014) 181(5):540-7. doi: 10.1667/RR13583.1

67. Wang L, Xie L, Tintani F, Xie H, Li C, Cui Z, et al. Aberrant Transforming Growth Factor- $\beta$ Activation Recruits Mesenchymal Stem Cells During Prostatic Hyperplasia. Stem Cells Transl Med (2017) 6(2):394-404. doi: 10.5966/sctm.2015-0411

68. Polz-Dacewicz M, Strycharz-Dudziak M, Dworzański J, Stec A, Kocot J. Salivary and Serum IL-10, TNF- $\alpha$, TGF- $\beta$, VEGF Levels in Oropharyngeal Squamous Cell Carcinoma and Correlation With HPV and EBV Infections. Infect Agent Cancer (2016) 11:45. doi: 10.1186/s13027-016-0093-6

69. Khwaja SS, Baker C, Haynes W, Spencer CR, Gay H, Thorstad W, et al. High E6 Gene Expression Predicts for Distant Metastasis and Poor Survival in Patients With HPV-Positive Oropharyngeal Squamous Cell Carcinoma. Int J Radiat Oncol Biol Phys (2016) 95(4):1132-41. doi: 10.1016/j.ijrobp.2016.03.001

70. Mariathasan S, Turley SJ, Nickles D, Castiglioni A, Yuen K, Wang Y, et al. Tgf $\beta$ Attenuates Tumour Response to PD-L1 Blockade by Contributing to Exclusion of T Cells. Nature (2018) 554(7693):544-8. doi: 10.1038/nature25501

71. Ganesh K, Massagué J. TGF- $\beta$ Inhibition and Immunotherapy: Checkmate. Immunity (2018) 48(4):626-8. doi: 10.1016/j.immuni.2018.03.037

72. Heeren AM, Rotman J, Stam AGM, Pocorni N, Gassama AA, Samuels S, et al. Efficacy of PD-1 Blockade in Cervical Cancer Is Related to a CD8+FoxP3(+)CD25(+) T-Cell Subset With Operational Effector Functions Despite High Immune Checkpoint Levels. J Immunother Cancer (2019) 7(1):43. doi: 10.1186/s40425-019-0526-Z

73. Brunen D, Willems SM, Kellner U, Midgley R, Simon I, Bernards R. TGFBeta: An Emerging Player in Drug Resistance. Cell Cycle (2013) 12(18):29608. doi: $10.4161 /$ cc. 26034

74. Marchini S, Fruscio R, Clivio L, Beltrame L, Porcu L, Fuso Nerini I, et al. Resistance to Platinum-Based Chemotherapy Is Associated With Epithelial to Mesenchymal Transition in Epithelial Ovarian Cancer. Eur J Cancer (2013) 49(2):520-30. doi: 10.1016/j.ejca.2012.06.026

75. Holmgaard RB, Schaer DA, Li Y, Castaneda SP, Murphy MY, Xu X, et al. Targeting the TGF $\beta$ Pathway With Galunisertib, a TGFbetaRI Small Molecule Inhibitor, Promotes Anti-Tumor Immunity Leading to Durable, Complete Responses, as Monotherapy and in Combination With Checkpoint Blockade. J Immunother Cancer (2018) 6(1):47. doi: 10.1186/ s40425-018-0356-4

76. Tauriello DVF, Palomo-Ponce S, Stork D, Berenguer-Llergo A, BadiaRamentol J, Iglesias M, et al. TGF $\beta$ Drives Immune Evasion in Genetically Reconstituted Colon Cancer Metastasis. Nature (2018) 554(7693):538-43. doi: 10.1038/nature25492

77. Principe DR, Park A, Dorman MJ, Kumar S, Viswakarma N, Rubin J, et al. TGF $\beta$ Blockade Augments PD-1 Inhibition to Promote T-Cell-Mediated Regression of Pancreatic Cancer. Mol Cancer Ther (2019) 18(3):613-20. doi: 10.1158/1535-7163.MCT-18-0850

78. Horn LA, Riskin J, Hempel HA, Fousek K, Lind H, Hamilton DH, et al. Simultaneous Inhibition of CXCR1/2, TGF- $\beta$, and PD-L1 Remodels the Tumor and Its Microenvironment to Drive Antitumor Immunity. J Immunother Cancer (2020) 8(1). doi: 10.1136/jitc-2019-000326

79. Zhu H, Gu X, Xia L, Zhou Y, Bouamar H, Yang J, et al. A Novel TGF $\beta$ Trap Blocks Chemotherapeutics-Induced TGF $\beta 1$ Signaling and Enhances Their Anticancer Activity in Gynecologic Cancers. Clin Cancer Res (2018) 24 (12):2780-93. doi: 10.1158/1078-0432.CCR-17-3112

80. Biswas S, Guix M, Rinehart C, Dugger TC, Chytil A, Moses HL, et al. Inhibition of TGF-Beta With Neutralizing Antibodies Prevents RadiationInduced Acceleration of Metastatic Cancer Progression. J Clin Invest (2007) 117(5):1305-13. doi: 10.1172/JCI30740

81. Gong J, Le TQ, Massarelli E, Hendifar AE, Tuli R. Radiation Therapy and PD-1/PD-L1 Blockade: The Clinical Development of an Evolving Anticancer Combination. J Immunother Cancer (2018) 6(1):46. doi: 10.1186/s40425018-0361-7

82. Bailly C, Thuru X, Quesnel B. Combined Cytotoxic Chemotherapy and Immunotherapy of Cancer: Modern Times. NAR Cancer (2020) 2(1). doi: 10.1093/narcan/zcaa002

83. Bhola NE, Balko JM, Dugger TC, Kuba MG, Sánchez V, Sanders M, et al. TGF- $\beta$ Inhibition Enhances Chemotherapy Action Against Triple-Negative Breast Cancer. J Clin Invest (2013) 123(3):1348-58. doi: 10.1172/JCI65416 
84. Dancea HC, Shareef MM, Ahmed MM. Role of Radiation-Induced TGFBeta Signaling in Cancer Therapy. Mol Cell Pharmacol (2009) 1(1):44-56. doi: $10.4255 / \mathrm{mcpharmacol} .09 .06$

85. Löffek S. Transforming of the Tumor Microenvironment: Implications for TGF- $\beta$ Inhibition in the Context of Immune-Checkpoint Therapy. J Oncol (2018) 2018:9732939. doi: 10.1155/2018/9732939

86. Meng Y, Liang H, Hu J, Liu S, Hao X, Wong MSK, et al. PD-L1 Expression Correlates With Tumor Infiltrating Lymphocytes and Response to Neoadjuvant Chemotherapy in Cervical Cancer. J Cancer (2018) 9 (16):2938-45. doi: 10.7150/jca.22532

87. Oaknin A, Gil-Martin M, Diver E, Jehl G, Gleicher SA, Chaudhary S, et al. Phase 1b Trial of First-Line Bintrafusp Alfa, a Bifunctional Fusion Protein Targeting TGF- $\beta$ and PD-L1, Plus Chemotherapy With or Without Bevacizumab in Cervical Cancer. Int J Gynecol Cancer (2021) 31(suppl 3):917. doi: doi: 10.1136/ijgc-2021-ESGO.75

88. Melief CJM, Welters MJP, Vergote I, Kroep JR, Kenter GG, Ottevanger PB, et al. Strong Vaccine Responses During Chemotherapy Are Associated With Prolonged Cancer Survival. Sci Transl Med (2020) 12(535). doi: 10.1126/ scitranslmed.aaz8235

89. Massarelli E, William W, Johnson F, Kies M, Ferrarotto R, Guo M, et al. Combining Immune Checkpoint Blockade and Tumor-Specific Vaccine for Patients With Incurable Human Papillomavirus 16-Related Cancer: A Phase 2 Clinical Trial. JAMA Oncol (2019) 5(1):67-73. doi: 10.1001/ jamaoncol.2018.4051

90. Naumann RW, Hollebecque A, Meyer T, Devlin MJ, Oaknin A, Kerger J, et al. Safety and Efficacy of Nivolumab Monotherapy in Recurrent or Metastatic Cervical, Vaginal, or Vulvar Carcinoma: Results From the Phase I/II CheckMate 358 Trial. J Clin Oncol (2019) 37(31):2825-34. doi: 10.1200/JCO.19.00739

91. Naumann RW, Oaknin A, Meyer T, Lopez-Picazo JM, Lao C, Bang Y, et al. Efficacy and Safety of Nivolumab (Nivo) + Ipilimumab (Ipi) in Patients (Pts) With Recurrent/Metastatic (R/M) Cervical Cancer: Results From CheckMate 358. Ann Oncol (2019) 30(suppl 5):Abstract 5630. doi: 10.1093/annonc/mdz394.059

92. Pena A. Early Data Support Agenus' 2 Lead Immunotherapies for Advanced Cervical Cancer. In: Immuno-Oncology News. (2020). Available at: https:// immuno-oncologynews.com/2020/03/04/balstilimab-zalifrelimab-andagen1181-show-early-signs-of-efficacy-and-safety-in-advanced-cervicalcancer-and-other-solid-tumors/ (Accessed September 30, 2021).

93. O'Malley DM, Oaknin A, Monk BJ, Selle F, Rojas C, Gladieff L, et al. Phase II Study of the Safety and Efficacy of the Anti-PD-1 Antibody Balstilimab in Patients With Recurrent and/or Metastatic Cervical Cancer. Gynecol Oncol (2021) 163(2):274-80. doi: 10.1016/j.ygyno.2021.08.018

94. Strauss J, Braiteh F, Calvo E, de Miguel MJ, Cervantes A, Edenfield WJ, et al. Evaluation of Bintrafusp Alfa, a Bifunctional Fusion Protein Targeting TGF$\beta$ and PD-L1, in Cervical Cancer: Data From Phase 1 and Phase 2 Studies. J Clin Oncol (2021) 39(15 suppl):Abstract 5509. doi: 10.1200/JCO. 2021.39.15_suppl.5509

95. Choi CH, Kim B-G, Lee J-W, Bae D-S, Kang E-S, Kim Y-M, et al. Phase IIa Study of BVAC-C in HPV Type 16 or 18 Positive Recurrent Cervical Carcinoma. J Clin Oncol (2021) 39(15 suppl):Abstract 5512. doi: 10.1200/ JCO.2021.39.15_suppl.5512

96. Tewari K, Monk B, Vergote I, Miller A, De Melo AC, Kim HS, et al. EMPOWER-Cervical 1/GOG-3016/ENGOT-Cx9: Results of Phase 3 Trial of Cemiplimab vs Investigator's Choice Chemotherapy in Recurrent/Metastatic Cervical Carcinoma. Int J Gynecol Cancer (2021) 31(suppl 4). doi: doi: 10.1136/ijgc-2021-IGCS.1

97. Jazaeri AA, Zsiros E, Amaria RN, Artz AS, Edwards RP, Wenham RM, et al. Safety and Efficacy of Adoptive Cell Transfer Using Autologous Tumor Infiltrating Lymphocytes (LN-145) for Treatment of Recurrent, Metastatic, or Persistent Cervical Carcinoma. J Clin Oncol (2019) 37(15 suppl):2538-8. doi: 10.1200/JCO.2019.37.15_suppl.2538

98. Santin AD, Deng W, Frumovitz M, Buza N, Bellone S, Huh W, et al. Phase II Evaluation of Nivolumab in the Treatment of Persistent or Recurrent Cervical Cancer (NCT02257528/NRG-Gy002). Gynecol Oncol (2020) 157 (1):161-6. doi: 10.1016/j.ygyno.2019.12.034

99. Frenel JS, Le Tourneau C, O'Neil B, Ott PA, Piha-Paul SA, Gomez-Roca C, et al. Safety and Efficacy of Pembrolizumab in Advanced, Programmed
Death Ligand 1-Positive Cervical Cancer: Results From the Phase Ib KEYNOTE-028 Trial. J Clin Oncol (2017) 35(36):4035-41. doi: 10.1200/ JCO.2017.74.5471

100. Wu X, Xia L, Zhou Q, Zhu J, Wang K, Chen J, et al. 357 GLS-010 (Zimberelimab), A Novel Fully Human Anti-PD-1 mAb in Chinese Patients With Recurrent/Metastatic Cervical Cancer: Results From a Multicenter, Open-Label, Single-Arm Phase II Trial. Int J Gynecol Cancer (2020) 30(Suppl 3):A147. doi: 10.1136/ijgc-2020-IGCS.307

101. Friedman CF, Snyder Charen A, Zhou Q, Carducci MA, Buckley De Meritens A, Corr BR, et al. Phase II Study of Atezolizumab in Combination With Bevacizumab in Patients With Advanced Cervical Cancer. J Immunother Cancer (2020) 8(2). doi: 10.1136/jitc-2020-001126

102. O'Malley DM, Neffa M, Monk BJ, Melkadze T, Huang M, Kryzhanivska A, et al. Dual PD-1 and CTLA-4 Checkpoint Blockade Using Balstilimab and Zalifrelimab Combination as Second-Line Treatment for Advanced Cervical Cancer: An Open-Label Phase II Study. J Clin Oncol (2021). doi: 10.1200/ JCO.21.02067

103. Lan C, Shen J, Wang Y, Li J, Liu Z, He M, et al. Camrelizumab Plus Apatinib in Patients With Advanced Cervical Cancer (CLAP): A Multicenter, OpenLabel, Single-Arm, Phase II Trial. J Clin Oncol (2020) 38(34):4095-106. doi: 10.1200/JCO.20.01920

104. Callahan MK, Odunsi K, Sznol M, Nemunaitis JJ, Ott PA, Dillon PM, et al. Phase 1 Study to Evaluate the Safety and Tolerability of MEDI4736 (Durvalumab, DUR) + Tremelimumab (TRE) in Patients With Advanced Solid Tumors. J Clin Oncol (2017) 35(15 suppl):Abstract 3069. doi: 10.1200/ JCO.2017.35.15_suppl.3069

105. Park JS, Hur S-Y, Lim MC, Kim YM, No JH, Kim B-G, et al. Efficacy and Safety Results of GX-188E, a Therapeutic DNA Vaccine, Combined With Pembrolizumab Administration in Patients With HPV 16- and/or 18Positive Advanced Cervical Cancer: Phase II Interim Analysis Results (KEYNOTE-567). J Clin Oncol (2021) 39(15 suppl):Abstract 5511. doi: 10.1200/JCO.2021.39.15_suppl.5511

106. Italiano A, Verlingue L, Prenen H, Guerra EM, Tosi D, Perets R, et al. Clinical Activity and Safety of Simlukafusp Alfa, an Engineered Interleukin-2 Variant Targeted to Fibroblast Activation Protein- $\alpha$, Combined With Atezolizumab in Patients With Recurrent or Metastatic Cervical Cancer. J Clin Oncol (2021) 39:(suppl 15; abstr 5510). doi: 10.1200/JCO. 2021.39.15_suppl.5510

107. Xu Q, Chen C, Sun Y, Huang Z, Lin Y, Liu J, et al. Anlotinib Plus Sintilimab in Patients With Recurrent Advanced Cervical Cancer: A Prospective, Multicenter, Single-Arm, Phase II Clinical Trial. J Clin Oncol (2021) 39, 2021:suppl 15; abstr 5524. doi: 10.1200/JCO.2021.39.15_suppl.5524

108. Grau JF, Farinas-Madrid L, Oaknin A. A Randomized Phase III Trial of Platinum Chemotherapy Plus Paclitaxel With Bevacizumab and Atezolizumab Versus Platinum Chemotherapy Plus Paclitaxel and Bevacizumab in Metastatic (Stage IVB), Persistent, or Recurrent Carcinoma of the Cervix: The BEATcc Study (ENGOT-Cx10/GEICO 68C/JGOG1084/GOG-3030). Int J Gynecol Cancer (2020) 30(1):139-43. doi: 10.1136/ijgc-2019-000880

109. Strauss J, Gatti-Mays ME, Cho BC, Hill A, Salas S, McClay E, et al. Bintrafusp Alfa, a Bifunctional Fusion Protein Targeting TGF- $\beta$ and PD-L1, in Patients With Human Papillomavirus-Associated Malignancies. J Immunother Cancer (2020) 8(2). doi: 10.1136/jitc-2020-001395

110. Diaz JP, Duan W, Schroeder E, Diaz Z, Lambrou N, Gatcliffe TA, et al. Immunotherapy in Combination With PARP Inhibition in Advanced Cervical Cancer Patients Functionally Competent or Deficient for the Fanconi Anemia Repair Pathway. J Clin Oncol (2021) 39(2021):(suppl 15; abstr TPS5597). doi: 10.1200/JCO.2021.39.15_suppl.TPS5597

111. Wu L, Wang P-H, Hsiao S-Y, Chang C-L, Kim HS, Lee J-Y, et al. AdvanTIG202: A Phase 2 Study Investigating Anti-TIGIT Monoclonal Antibody Ociperlimab Plus Anti-PD-1 Monoclonal Antibody Tislelizumab in Patients With Previously Treated Recurrent or Metastatic Cervical Cancer. J Clin Oncol (2021) 39(2021):suppl 15; abstr TPS5595. doi: 10.1200/ JCO.2021.39.15_suppl.TPS5595

112. Welters MJ, van der Sluis TC, van Meir H, Loof NM, van Ham VJ, van Duikeren S, et al. Vaccination During Myeloid Cell Depletion by Cancer Chemotherapy Fosters Robust T Cell Responses. Sci Transl Med (2016) 8 (334):334ra52. doi: 10.1126/scitranslmed.aad8307 
113. Regeneron. Phase 3 Trial of Libtayo ${ }^{\circledR}$ (Cemiplimab) Monotherapy in Advanced Cervical Cancer Stopped Early for Positive Result on Overall Survival. News Release. Available at: https://newsroom.regeneron.com/ news-releases/news-release-details/phase-3-trial-libtayor-cemiplimabmonotherapy-advanced-cervical (Accessed September 30, 2021).

114. Kojima A, Mikami Y, Sudo T, Yamaguchi S, Kusanagi Y, Ito M, et al. Gastric Morphology and Immunophenotype Predict Poor Outcome in Mucinous Adenocarcinoma of the Uterine Cervix. Am J Surg Pathol (2007) 31(5):66472. doi: 10.1097/01.pas.0000213434.91868.b0

115. Santin A, Deng W, Frumovitz MW, Huh WK, Khleif S, Lankes HA, et al. A Phase II Evaluation of Nivolumab, a Fully Human Antibody Against PD-1, in the Treatment of Persistent or Recurrent Cervical Cancer. J Clin Oncol (2018) 36(15 suppl):Abstract 5536. doi: 10.1200/JCO.2018.36.15_suppl.5536

116. Lacal PM, Tentori L, Muzi A, Ruffini F, Dorio AS, Xu W, et al. Pharmacological Inhibition of Poly(ADP-Ribose) Polymerase Activity Down-Regulates the Expression of Syndecan-4 and Id-1 in Endothelial Cells. Int J Oncol (2009) 34(3):861-72. doi: 10.3892/ijo_00000213

117. Tentori L, Lacal PM, Muzi A, Dorio AS, Leonetti C, Scarsella M, et al. Poly (ADP-Ribose) Polymerase (PARP) Inhibition or PARP-1 Gene Deletion Reduces Angiogenesis. Eur J Cancer (2007) 43(14):2124-33. doi: 10.1016/ j.ejca.2007.07.010

118. Shen J, Zhao W, Ju Z, Wang L, Peng Y, Labrie M, et al. PARPi Triggers the STING-Dependent Immune Response and Enhances the Therapeutic Efficacy of Immune Checkpoint Blockade Independent of BRCAness. Cancer Res (2019) 79(2):311-9. doi: 10.1158/0008-5472.CAN-18-1003

119. Huang J, Wang L, Cong Z, Amoozgar Z, Kiner E, Xing D, et al. The PARP1 Inhibitor BMN 673 Exhibits Immunoregulatory Effects in a $\mathrm{Brcal}^{-/-}$Murine Model of Ovarian Cancer. Biochem Biophys Res Commun (2015) 463(4):5516. doi: 10.1016/j.bbrc.2015.05.083

120. Pyriochou A, Olah G, Deitch EA, Szabó C, Papapetropoulos A. Inhibition of Angiogenesis by the Poly(ADP-Ribose) Polymerase Inhibitor PJ-34. Int $J$ Mol Med (2008) 22(1):113-8. doi: 10.3892/ijmm.22.1.113

121. Lucarini L, Durante M, Lanzi C, Pini A, Boccalini G, Calosi L, et al. HYDAMTIQ, A Selective PARP-1 Inhibitor, Improves Bleomycin-Induced Lung Fibrosis by Dampening the TGF- $3 /$ SMAD Signalling Pathway. J Cell Mol Med (2017) 21(2):324-35. doi: 10.1111/jcmm.12967

122. Krishnan V, Chong YL, Tan TZ, Kulkarni M, Bin Rahmat MB, Tay LS, et al. TGF $\beta$ Promotes Genomic Instability After Loss of RUNX3. Cancer Res (2018) 78(1):88-102. doi: 10.1158/0008-5472.CAN-17-1178

123. Lan Y, Zhang D, Xu C, Hance KW, Marelli B, Qi J, et al. Enhanced Preclinical Antitumor Activity of M7824, A Bifunctional Fusion Protein Simultaneously Targeting PD-L1 and TGF- $\beta$. Sci Transl Med (2018) 10(424). doi: 10.1126/ scitranslmed.aan 5488

124. Strauss J, Heery CR, Schlom J, Madan RA, Cao L, Kang Z, et al. Phase I Trial of M7824 (MSB0011359C), A Bifunctional Fusion Protein Targeting PD-L1 and TGF $\beta$, in Advanced Solid Tumors. Clin Cancer Res (2018) 24(6):128795. doi: 10.1158/1078-0432.CCR-17-2653

125. Knudson KM, Hicks KC, Luo X, Chen JQ, Schlom J, Gameiro SR. M7824, A Novel Bifunctional Anti-PD-L1/TGF $\beta$ Trap Fusion Protein, Promotes AntiTumor Efficacy as Monotherapy and in Combination With Vaccine. Oncoimmunology (2018) 7(5):e1426519. doi: 10.1080/2162402X.2018. 1426519

126. Dodagatta-Marri E, Meyer DS, Reeves MQ, Paniagua R, To MD, Binnewies $\mathrm{M}$, et al. $\alpha$-PD-1 Therapy Elevates Treg/Th Balance and Increases Tumor Cell Psmad 3 That Are Both Targeted by $\alpha$-TGF $\beta$ Antibody to Promote Durable Rejection and Immunity in Squamous Cell Carcinomas. J Immunother Cancer (2019) 7(1):62. doi: 10.1186/s40425-018-0493-9

127. Allan S, Braiteh F, Aller E, Cervantes A, Edenfield W, Li T, et al. P37 Phase 1 Evaluation of Bintrafusp Alfa (M7824), a Bifunctional Fusion Protein Targeting TGF- $\beta$ and PD-L1, in Cervical Cancer. Int J Gynecol Cancer (2019) 29(suppl 4):Abstract P37. doi: 10.1136/ijgc-2019-ESGO.100
128. Gulley J, Strauss J, Gatti-Mays ME, Cho BC, Hill A, Salas S, et al. Long-Term Follow-Up of Patients With Human Papillomavirus (HPV)-associated Malignancies Treated With Bintrafusp Alfa, a Bifunctional Fusion Protein Targeting TGF- $\beta$ and PD-L1. Ann Oncol (2021) 32(suppl 5):Abstract 9570. doi: 10.1016/annonc/annonc705

129. Ni Y, Soliman A, Joehlin-Price A, Rose PG, Vlad A, Edwards RP, et al. High TGF- $\beta$ Signature Predicts Immunotherapy Resistance in Gynecologic Cancer Patients Treated With Immune Checkpoint Inhibition. NPJ Precis Oncol (2021) 5(1):101. doi: 10.1038/s41698-021-00242-8

130. Chan TA, Yarchoan M, Jaffee E, Swanton C, Quezada SA, Stenzinger A, et al. Development of Tumor Mutation Burden as an Immunotherapy Biomarker: Utility for the Oncology Clinic. Ann Oncol (2019) 30(1):44-56. doi: 10.1093/ annonc/mdy 495

Conflict of Interest: KF reports institutional research funding from Merck and MSD. AO reports institutional research funding from AbbVie Deutschland, Abililty Pharmaceuticals, Advaxis, Aeterna Zentaris, Amgen SA, Aprea Therapeutics AB, Bristol Myers Squibb, Clovis Oncology, Eisai, Immunogen, Millennium Pharmaceuticals, MSD de España SA, Pharmamar SA, Regeneron Pharmaceuticals, Roche, and Tesaro; served on advisory boards for AstraZeneca Farmacéutica Spain SA, AstraZeneca KK, Clovis Oncology, Corcept Therapeutics, Deciphera Pharmaceuticals, Eisai, Merck, Roche, GlaxoSmithKline, Got It Consulting SL, Immunogen, KL Logistics, Medison Pharma, MSD de España, Mersana Therapeutics, Novocure GmbH, Pharmamar SA, prIME Oncology, Roche Farma, Shattuck Labs, Sutro Biopharma, Tesaro Bio GmbH, Tesaro Bio Spain SL, and Tesaro; and received travel or accommodation expenses from AstraZeneca, Pharmamar, and Roche. LR received honoraria from BluePrint Oncology, CurioScience, Physicians' Education Research, and Products in Knowledge; reports consulting or advisory role for Agenus, AstraZeneca, Clovis Oncology, Merck, Genentech/Roche, GOG Foundation, MSD, Mersana, Myriad Genetics, Novartis, Rubius Therapeutics, and Seagen; participated in speakers bureau for AstraZeneca, MSD, and Tesaro; and reports institutional research funding from Aivita Biomedical, Akeso Biopharma, AstraZeneca, GEICO, Genentech/Roche, MSD, On Target Laboratories, Pfizer, and Tesaro. LO reports employment, at the time of the study, with EMD Serono Research \& Development Institute, Inc, Billerica, MA, USA, an affiliate of Merck KGaA. CV reports employment with Merck.

The remaining authors declare that the research was conducted in the absence of any commercial or financial relationships that could be construed as a potential conflict of interest.

The authors declare that this work was funded by Merck (CrossRef Funder ID: 10.13039/100009945) and was previously part of an alliance between Merck and GlaxoSmithKline. The funder had the following involvement with the study: two authors (L.S. Ojalvo and C. Valencia) were employees of EMD Serono Research \& Development Institute, Inc, Billerica, MA, USA, an affiliate of Merck KGaA.

Publisher's Note: All claims expressed in this article are solely those of the authors and do not necessarily represent those of their affiliated organizations, or those of the publisher, the editors and the reviewers. Any product that may be evaluated in this article, or claim that may be made by its manufacturer, is not guaranteed or endorsed by the publisher.

Copyright (c) 2022 Birrer, Fujiwara, Oaknin, Randall, Ojalvo, Valencia and RayCoquard. This is an open-access article distributed under the terms of the Creative Commons Attribution License (CC BY). The use, distribution or reproduction in other forums is permitted, provided the original author(s) and the copyright owner(s) are credited and that the original publication in this journal is cited, in accordance with accepted academic practice. No use, distribution or reproduction is permitted which does not comply with these terms. 